\title{
Wavelet-based Gaussian-mixture hidden Markov model for the detection of multistage seizure dynamics: A proof-of-concept study
}

\author{
Alan WL Chiu ${ }^{1 *}$, Miron Derchansky², Marija Cotic ${ }^{2,3}$, Peter L Carlen², Steuart O Turner ${ }^{1}$ and Berj L Bardakjian ${ }^{3}$
}

\author{
* Correspondence: \\ alanchiu@latech.edu \\ ${ }^{1}$ Biomedical Engineering \\ Department, Louisiana Tech \\ University, Ruston, Louisiana, USA \\ Full list of author information is \\ available at the end of the article
}

\begin{abstract}
Background: Epilepsy is a common neurological disorder characterized by recurrent electrophysiological activities, known as seizures. Without the appropriate detection strategies, these seizure episodes can dramatically affect the quality of life for those afflicted. The rationale of this study is to develop an unsupervised algorithm for the detection of seizure states so that it may be implemented along with potential intervention strategies.

Methods: Hidden Markov model (HMM) was developed to interpret the state transitions of the in vitro rat hippocampal slice local field potentials (LFPS) during seizure episodes. It can be used to estimate the probability of state transitions and the corresponding characteristics of each state. Wavelet features were clustered and used to differentiate the electrophysiological characteristics at each corresponding HMM states. Using unsupervised training method, the HMM and the clustering parameters were obtained simultaneously. The HMM states were then assigned to the electrophysiological data using expert guided technique. Minimum redundancy maximum relevance (mRMR) analysis and Akaike Information Criterion (AICc) were applied to reduce the effect of over-fitting. The sensitivity, specificity and optimality index of chronic seizure detection were compared for various HMM topologies. The ability of distinguishing early and late tonic firing patterns prior to chronic seizures were also evaluated.
\end{abstract}

Results: Significant improvement in state detection performance was achieved when additional wavelet coefficient rates of change information were used as features. The final HMM topology obtained using mRMR and AICc was able to detect non-ictal (interictal), early and late tonic firing, chronic seizures and postictal activities. A mean sensitivity of $95.7 \%$, mean specificity of $98.9 \%$ and optimality index of 0.995 in the detection of chronic seizures was achieved. The detection of early and late tonic firing was validated with experimental intracellular electrical recordings of seizures.

Conclusions: The HMM implementation of a seizure dynamics detector is an improvement over existing approaches using visual detection and complexity measures. The subjectivity involved in partitioning the observed data prior to training can be eliminated. It can also decipher the probabilities of seizure state transitions using the magnitude and rate of change wavelet information of the LFPs. 


\section{Background}

Epilepsy is one of the most common neurological disorders, affecting over 50 million people worldwide. The disorder is characterized by spontaneous, recurrent, seemingly unpredictable symptoms called seizures [1,2]. A seizure can be defined as the sudden manifestation of lowered complexity synchronized rhythmic activities across populations of neurons [3]. Epilepsy affects motor and speech, as well as other cognitive functions that, if untreated, can lead to permanent damage to the brain. Time-frequency representation (TFR) is an important marker for understanding the progression into seizure onsets [4]. The progression to the seizure onset is associated with the entrainment of neuronal population firing. In particular, the TFR of epileptiform oscillation has been suggested as a useful tool in localizing regions of seizure onsets and in understanding the mechanisms behind seizure generation [5]. The current methods for detecting the state transitions of seizure episodes based on TFR usually involve the computation of state-specific features through supervised learning techniques [6-10]. In general, supervised learning strategies require certain knowledge of the system so that the data can be separated into different states based on their known dynamics before the training process. To date, a great deal of subjectivity is required for the implementation of these algorithms since the desired solution for detection must be defined by the experimenter.

Seizure detection refers to the identification of seizure onsets a few seconds before the observable behavioral changes [11,12]. Several effective supervised pattern recognition strategies have been developed for seizure detection. Multi-layered networks were first introduced to analyze EEG data pertaining to seizure phenomenon in the mid1990s [13,14]. Various methods such as autoregressive models $[15,16]$ to more advanced techniques such as support vector machines $[17,18]$ were also proposed. The feature space for these approaches ranged from spectrogram [19], dominant frequency, power and amplitude [20] to time frequency distribution [21] such as wavelet transform based approximate entropy [22,23]. It has been reported that frequency content of neuronal electrical activity changed significantly during the progression of a seizure, both in the interspike interval and in the intraburst dynamics $[8,24]$. The major disadvantage of these supervised methods is that their performances can only be as good as the initial data separation criteria. Therefore, it is essential to utilize an unsupervised learning paradigm that transcends these restrictions.

An unsupervised probabilistic approach for the detection of seizure-like events (SLEs) in vitro extracellular local field potentials (LFPs) seizures using hidden Markov model (HMM) [25,26] along with clustering of wavelet features is proposed. The in vitro model, emulating human epilepsy, provides a platform for testing the seizure dynamics detection algorithm. The HMM is not meant to reproduce the exact electrophysiological recordings of the brain. Instead, it is used to capture the essential TFR characteristics in the progression of SLEs and to estimate the state transitions as a multi-stage process. The HMM has an advantage over the supervised approaches because it does not require prior manual separation of data into different dynamics. The current approach of detecting seizure events using Markov models involves the estimation of either two (seizure and interictal) [27] or three (baseline, detected and seizure) [28] distinct states. Even though these proposed methods appear to detect seizure onsets, they failed to address the possibility of having multiple distinctive dynamics between non-ictal (interictal) and chronic seizure events, which may be an 
important aspect for the development of seizure therapy techniques. The training process of the HMM is an unsupervised approach. However, a certain amount of bias can be achieved by making an informed choice on the assignment of the model states to the electrographical activities after the unsupervised training process is completed.

To determine the optimal HMM topology for seizure detection, two methods were proposed and compared. First, the performance of the trained HMM was evaluated on the validation set to determine the suitable HMM topology. Second, minimum redundancy maximum relevance (mRMR) analysis [29] and Akaike Information Criteria (AICc) $[30,31]$ were used to find a suitable feature space and optimum model by balancing the log-likelihood (LL) against the number of model parameters. Furthermore, the performances of HMMs were compared with the wavelet-based supervised machine learning techniques [6,7] based on the sensitivity and specificity of chronic seizure detection, the detection delay and the optimality measure [32]. We hypothesized that using appropriate wavelet features, the HMM can detect the various stages of SLEs at least as well as, if not better than, the supervised machine learning algorithms. We also hypothesized that the optimal HMM topology can illuminate multiple transitional characteristics in the tonic firing phrase leading to the onsets of chronic seizure activities.

\section{Methods}

\section{A. Tissue preparation and data acquisition}

Hippocampal slice recordings were obtained from eight Wistar rats (17-25 days old). The animals were anaesthetized with halothane and decapitated in accordance with the Canadian Animal Care Guidelines. The brains were promptly dissected and maintained in ice-cold $\left(4^{\circ} \mathrm{C}\right)$ artificial cerebrospinal fluid (aCSF) for four to five minutes. Each brain was incised in a horizontal manner in accordance with the procedure outlined by [33,34]. The dorsal cortex of each hemisphere was cut parallel to the rostral/caudal axis and glued dorsal side down to an aluminium block, with caudal end towards the blade. The block was secured at a $12-14^{\circ}$ angle, and brain slices of $400 \mu \mathrm{m}$ thick were sectioned using a vibratome. Next, slices were maintained at room temperature in oxygenated "standard" aCSF $\left(95 \% \mathrm{O}_{2}, 5 \% \mathrm{CO}_{2}\right)$ for a minimum of one hour prior to recording. The composition of the "standard" aCSF was as follows (in $\mathrm{mM}$ ), $\mathrm{NaCl}$ (125), $\mathrm{KCl}$ (5), $\mathrm{NaH}_{2} \mathrm{PO}_{4}$ (1.25), $\mathrm{MgSO}_{4}$ (2), $\mathrm{CaCl}_{2}$ (2), $\mathrm{NaHCO}_{3}$ (25) and glucose (10). The $\mathrm{pH}$ was approximately 7.4, with osmolarity in the range of $300 \pm 5 \mathrm{mOsm}$. During the data acquisition stage, slices were transferred to the fusion chamber maintained at $30^{\circ} \mathrm{C}$ (Medical Systems Corp., Model PDMI-2, Harvard Apparatus, St. Laurent, Quebec, Canada). The pyramidal cells were visualized with an upright microscope (BX51, Olympus, Melville, NY, USA) using infrared imaging with differential interference contrast (IR-DIC) under $40 \times$ magnification (water-immersion objective) with an OLY150IR camera-video monitor unit (Olympus) [35]. Pyramidal cells were identified based on their characteristic spike frequency of $15.0 \pm 5 \mathrm{~Hz}$, their morphological features and the general location of the electrode placement. At the time of recording, spontaneous SLEs were induced by perfusing the slice with low- $\mathrm{Mg}^{2+}$ ACSF (containing in mM: $123 \mathrm{NaCl}, 5 \mathrm{KCl}, 1.5 \mathrm{CaCl} 2,0.25 \mathrm{MgSO} 4,25 \mathrm{NaHCO}, 1.2 \mathrm{NaH} 2 \mathrm{PO} 4$ and 15 glucose), or by tetanic stimulation of the CA3 region in "standard" aCSF, once every $10 \mathrm{~min}(80 \mathrm{~Hz}, 1$ second duration), using a Grass S44 stimulator (Grass Medical Instruments, West Warwick, Rhode Island). The reduction of extracellular $\mathrm{Mg}^{2+}$ 
concentration has long been known to enhance neuronal excitability by decreasing membrane surface charge screening and, thereby, facilitating the activation of inward currents in addition to increasing the synaptic excitation by unblocking the NMDA receptor [36]. The population dynamic of the LFPs were measured using an aCSF-filled borosilicate glass pipette located in stratus pyramidal of the CA1 region of the hippocampus. Data was acquired using a custom-made DC differential amplifier with a lowpass filter (corner frequency $400 \mathrm{~Hz}$ ), digitized at $1 \mathrm{kHz}$ by a Digidata 1322 (Axon Instruments, Union City, California). The whole-cell patch-clamp recordings were performed in the current clamp configuration using an Axopatch 200B amplifier (Axon Instruments, Union City, CA, USA). The whole-cell patch pipette solutions contained (contained in mM: $8 \mathrm{NaCl}, 0.001 \mathrm{CaCl}_{2}, 10 \mathrm{Na}$-Hepes, $5 \mathrm{KCl}, 140$ potassium gluconate, $1 \mathrm{MgCl}_{2}, 0.3 \mathrm{Na}$-GTP and $2 \mathrm{Na}-\mathrm{ATP}$ ). The perforated patch pipette solution (containing in mM: $50 \mathrm{KCl}, 2$ Hepes, 0.1 EGTA and $\leq 50 \mu \mathrm{g} / \mathrm{ml}$ gramicidin).

Overall, this study utilized 50 SLEs from 20 hippocampal slices in 8 rats with at least two SLEs recordings per rat. Each SLE was normalized in amplitude between -1 and +1. The DC components and the $60 \mathrm{~Hz}$ noise along with their harmonics were removed using FIR notch filtering. All of the analyses were performed using MATLAB (MathWorks, Natick, MA). The entire date set contained SLEs 50-379 s in duration (mean \pm standard deviation of $145 \pm 70 \mathrm{~s}$ ). A typical SLE was made up of the interictal period, followed by tonic firing and then chronic seizure, before returning to the interictal period through the postictal activity. The mean duration of the chronic seizure was $74 \mathrm{~s}$ with standard deviation of $32 \mathrm{~s}$. The duration of the tonic firing pattern lasted 0-301 s (mean \pm standard deviation of $49 \pm 48 \mathrm{~s}$ ). We also included over $30 \mathrm{~min}$ of non-ictal/interictal bursting (IB) activities to determine the false positive rates of the seizure detectors. Details of the dataset for analysis are given in Table 1.

\section{B. Wavelet-based hidden Markov model seizure detector}

The HMM is a powerful technique for the estimation and analysis of state transitions in any potentially multi-stage process. It is particularly useful in describing the progression of time-varying phenomenon in which the observed signals are emitted from the underlying dynamical states whose detailed generating mechanism is unknown or hidden. This is also important because the underlying dynamics of the brain is still relatively unknown.

\section{Feature space}

The LFPs recorded near the stratus pyramidal of the CA1 region of in vitro rat hippocampal slices were used to train the HMM. The continuous wavelet transform (CWT) [37-39] using Morlet mother wavelet $\psi(t)$ was performed to extract the features. We have noted from our previous study that the choice of mother wavelet does not affect

Table 1 Characteristics of the data set

\begin{tabular}{cc}
\hline Characteristics & \\
\hline Duration of EEG recording & $121 \mathrm{~m}$ \\
Number of seizures & 50 \\
Duration of non-ictal activity & $30 \mathrm{~m}$ \\
Mean \pm Stdev of tonic firing & $49.8 \pm 48.0 \mathrm{~s}$ \\
Mean \pm Stdev of chronic seizure & $73.6 \pm 32.4 \mathrm{~s}$ \\
Sampling rate & $1024 \mathrm{~Hz}$ \\
\hline
\end{tabular}


the pattern recognition system [6]. The modified wavelets $\psi_{s, l}(t)$, derived from a mother wavelet by a scaling factor $s$ and a translation factor $l$, is defined as:

$$
\psi_{s, l}(t)=\frac{1}{\sqrt{s}} \psi\left(\frac{t-l}{s}\right)
$$

The wavelet coefficients $(c)$ can be computed at each non-overlapping 1-second moving time window, as the correlation between the modified wavelet and the input data. For this study, seven frequency components (as illustrated in Table 2) denoting important physiologically-relevant frequency bands were obtained. We evaluated two feature spaces. The first feature set was made up of 7-D wavelet coefficient data, which was identical to the feature set used in the previous supervised seizure detection algorithm called the wavelet artificial neural networks (WANN) [6,7]. The second feature set was a 14-D vector consisted of the information from the first feature set and the rate of change information $\Delta c$, defined as the numerical difference of $c$ from the previous time window at each frequency band.

\section{HMM topology}

The HMM topology is defined by the number of states $(\mathrm{Q})$ in the HMM and the number of basis functions $(\mathrm{M})$ used to represent the feature space. The value $\mathrm{Q}$ can be interpreted as the potential number of distinct dynamics that may exist within a SLE. The value M can loosely be interpreted as the complexity of the feature for each possible model state.

The model parameters of the HMM help create two probability functions: the state transition probability and the emission probability. The parameter $a_{i j}$ is the probability of state transition from state $i$ to state $j$ where $i$ and $j$ can range from 1 to Q. The model state $j$ at time $t$ is denoted as $\left(S_{t}=j\right)$.

$$
a_{i j}=P\left(S_{t+1}=j \mid S_{t}=i\right)
$$

In this study, Q could go up to 10, allowing for the detection of potential multi-stage SLE processes. The transition of the model states was also assumed to follow a static probability distribution such that the transition probability was independent of time.

For the representation of the features space, an unsupervised Mixture of Gaussians (MoGs) clustering technique was used. The feature vectors in the D-dimensional space is denoted as $\boldsymbol{x}$, where $\mathrm{D}=7$ or 14 depending of the features. The multivariate Gaussian probability density measure is a function of the center $\mu_{k}$ (D by 1 in size) and covariance matrix $\Sigma_{k}$ (D by $\mathrm{D}$ in size). It is defined as:

$$
\phi\left(x, \mu_{k}, \Sigma_{k}\right)=\frac{1}{(2 \pi)^{\frac{\mathrm{D}}{2}}\left|\Sigma_{k}\right|^{\frac{1}{2}}} e^{-\left\{\frac{1}{2}\left(x-\mu_{k}\right)^{T} \Sigma_{k}{ }^{-1}\left(x-\mu_{k}\right)\right\}}
$$

Table 2 Feature space for the HMM

\begin{tabular}{cc}
\hline Bands & Frequency Range $(\mathbf{H z})$ \\
\hline Delta & $<4$ \\
Theta & $4-8$ \\
Alpha & $8-15$ \\
Beta & $15-40$ \\
Gamma & $40-100$ \\
Super gamma & $100-250$ \\
Fast ripple & $250-400$ \\
\hline
\end{tabular}

The wavelet coefficients were divided into seven frequency bands according to the accepted physiological ranges. 
The HMM was set up such that each state $j$ would have M number of clusters in the feature space. Initially, the Gaussian clusters were randomly scattered and a subset of the feature $\boldsymbol{x}$ was assigned to the $\mathrm{k}^{\text {th }}$ cluster in the hidden state $j$. The output probability function, called emission probability $b_{j}(\boldsymbol{x})$ can then be defined as:

$$
b_{j}(x)=\sum_{k=1}^{M} w_{j k} \phi\left(x, \mu_{j k}, \Sigma_{j k}\right)
$$

Here, the weighting factor for each cluster is denoted as $w_{j k}$. In order to interpret $b_{j}$ $(\boldsymbol{x})$ as true probability, the weighting factor needs to satisfy the following criteria:

$$
\sum_{k=1}^{M} w_{j k}=1
$$

In this paper, the number of clusters was allowed to vary from 1 to 5 . A schematic representation of a 3-state HMM based on a 2-D feature space is shown in Figure 1, illustrating how the transition probabilities and the emission probabilities can tell us about the progression of the model states and the representation of Gaussian clusters in the state space.

\section{Training and validation}

The training of HMM involved the process of maximizing the probability of fitting the distribution of the feature space through an iterative process of updating the model parameters [40]. Forty percent of the SLEs were randomly selected as the training set. For each HMM topology ( $\mathrm{Q}=1$ to 10 and $\mathrm{M}=1$ to 5$)$, the model parameters $\left(a_{i j}, w_{j k} \mu_{j k}\right.$ and $\Sigma_{j k}$ ) related to HMM transitions and clustering were randomly initialized and estimated in an iterative manner simultaneously. These parameters were updated according to the Expectation-Maximization (EM) algorithm [25]. At the Expectation step, the responsibility

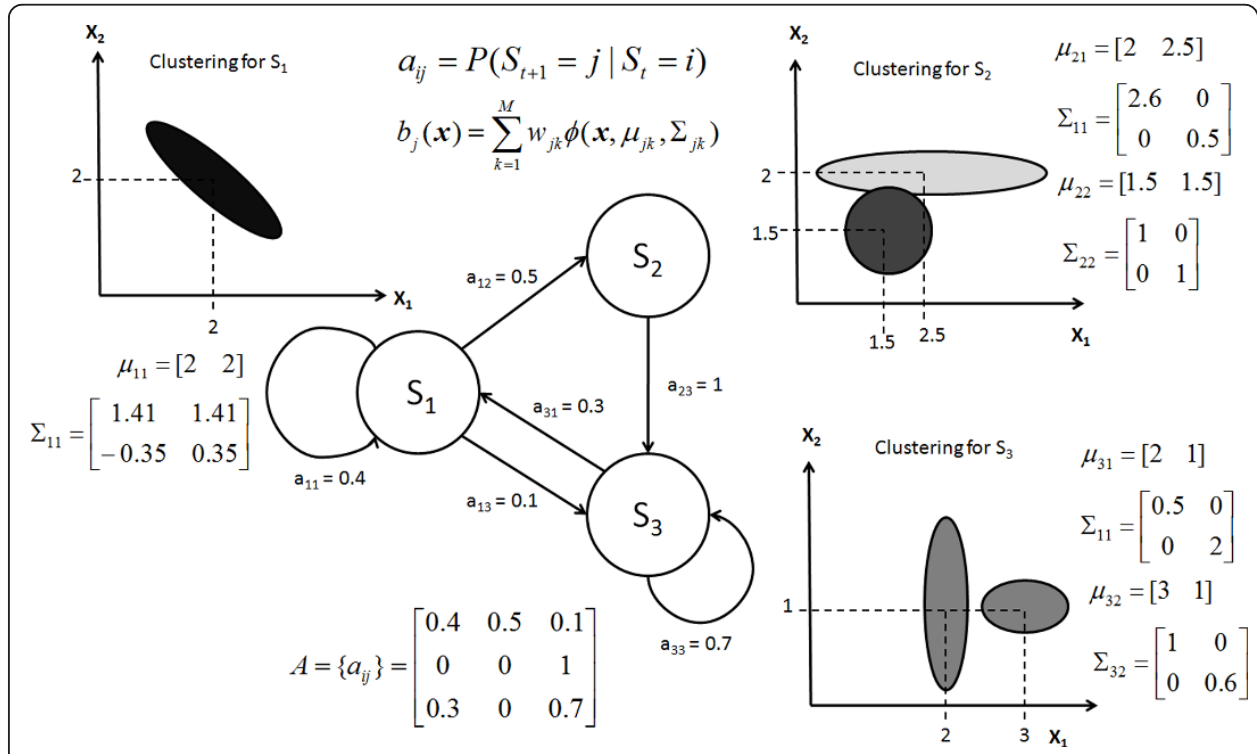

Figure 1 General schematic representation of hidden Markov model. A sample 3-state HMM with 2-D features space is illustrated. The state transition probably from state $i$ to $j$ is denoted by $a_{i j}$ and the emission probability of having feature $\boldsymbol{x}$ in state $j$ is denoted as $b_{j}(x)$. The mean $\mu_{j k}$ and covariance matrix $\Sigma_{j k}$ of each cluster provide information on the center and distribution of information in the feature space. 
of the model in representing the observed data was evaluated. The joint probability of observing all the data up to time $t$ at state $j$ was given as $\alpha_{j}(t)$ and the conditional probability of all the future data from time $t+1$ onward at state $i$ was given as $\beta_{i}(t)$. The joint probability and conditional probability can be updated in an iterative manner:

$$
\begin{aligned}
& \alpha_{j}(t+1)=\left[\sum_{i=1}^{Q} \alpha_{i}(t) a_{i j}\right] b_{j}\left(\boldsymbol{x}_{t+1}\right) \\
& \beta_{i}(t)=\sum_{j=1}^{Q} a_{i j} b_{j}\left(\boldsymbol{x}_{t+1}\right) \beta_{j}(t+1)
\end{aligned}
$$

Using $\alpha$ and $\beta$, the probability of $S_{t}=i$ is given by $\gamma_{i}(t)$. The probability of having $S_{t}$ $=i$ and $S_{t+1}=j$ is denoted as $\zeta_{i j}(t)$.

$$
\begin{aligned}
& \gamma_{i}(t)=\frac{\alpha_{i}(t) \beta_{i}(t)}{\sum_{j=1}^{Q} \alpha_{j}(t) \beta_{j}(t)} \\
& \zeta_{i j}(t)=\frac{\gamma_{i}(t) a_{i j} b_{j}\left(\boldsymbol{x}_{t+1}\right) \beta_{j}(t+1)}{\beta_{i}(t)}
\end{aligned}
$$

For the clustering of feature space using MoGs, the probability that the $\mathrm{k}^{\text {th }}$ cluster at state $j$ can generate a particular observation $\boldsymbol{x}_{\boldsymbol{t}}$ is given by:

$$
\gamma_{j k}(t)=\gamma_{j}(t) \frac{w_{j k} \phi\left(x_{t}, \mu_{j k}, \Sigma_{j k}\right)}{b_{j}\left(x_{t}\right)}
$$

From these estimates, the model parameters can be updated in the Maximization step.

$$
\begin{aligned}
a_{i j}^{\text {new }}= & \frac{\sum_{t=1}^{T-1} \gamma_{i j}(t)}{\sum_{t=1}^{T-1} \gamma_{i}(t)} \\
w_{j k}^{\text {new }}= & \frac{\sum_{t=1}^{T} \gamma_{j k}(t)}{\sum_{t=1}^{T} \gamma_{j}(t)} \\
\mu_{j k}^{\text {new }}= & \frac{\sum_{t=1}^{T} \gamma_{j k}(t) x_{t}}{\sum_{t=1}^{T} \gamma_{j k}(t)} \\
\sum_{j k}^{\text {new }}= & \frac{\sum_{t=1}^{T} \gamma_{j k}(t)\left(x_{t}-\mu_{j k}^{\text {new }}\right)\left(x_{t}-\mu_{j k}^{\text {new }}\right)^{T}}{\sum_{t=1}^{T} \gamma_{j k}(t)}
\end{aligned}
$$

The EM algorithm was repeated until the log-likelihood (LL) measure was no longer showing significant improvement. The LL measures quantified the goodness of fit between the feature $x$ :

$$
L L=\log \left(P\left(x \mid a_{i j}, w_{j k}, \mu_{j k}, \Sigma_{j k}\right)\right)
$$


The EM algorithm would typically take only a few seconds to complete. From the learning process described above, it should be obvious that if the number of clusters $(\mathrm{M})$ and the number of possible HMM states $(\mathrm{Q})$ were allowed to increase unrestricted, the LL would continue to improve at the expense of over-fitting the data.

Two methods were used to select the appropriate HMM model topology (Q and M) for each feature set. The first method involved the use of the validation data set. The performance of the trained HMMs at different $\mathrm{Q}$ and $\mathrm{M}$ combinations were examined on the validation sets consisting of $20 \%$ of the overall SLEs data. The HMM associated with the best overall balance between ictal and non-seizure data detection was denoted as the optimal HMMs. The optimal HMM obtained using 7-D wavelet coefficient features was denoted $\mathrm{HMM}_{\mathrm{opt} 7 \mathrm{D}}$ and the optimal HMM obtained using 14-D wavelet and rate of change features was denoted $\mathrm{HMM}_{\mathrm{opt14}}$. These models were then tested to evaluate the state transitions on the test data, consisting of the remaining $40 \%$ of the SLEs. The second method to find the HMM topology involved the use of minimum redundancy maximum relevance (mRMR) analysis [41] to reducing the dimensionality of the feature space, and the use of AICc [30]. The mRMR method, which has been frequently used in gene expression research, can identify subsets of feature that were relevant to the classification tasks. It selected the features that were mutually far apart from each other (small $W_{c}$ ) while still having a strong correlation (large $V_{F}$ ) to the target state.

$$
\begin{aligned}
& \max \quad V_{F}, \quad V_{F}=\frac{1}{|\Pi|} \sum_{f_{m} \in S} F\left(f_{m}, S\right) \\
& \min \quad W_{c}, \quad W_{c}=\frac{1}{|\Pi|^{2}} \sum_{m, n} C\left(f_{m}, f_{n}\right)
\end{aligned}
$$

Here, $\Pi$ represents the set of features, $C\left(f_{m} f_{n}\right)$ is the correlation between the two wavelet features $f_{m}$ and $f_{n}$ and $F\left(f_{m} S\right)$ is the F-statistics between the feature $f_{m}$ and the target chronic seizure state $S$. The wavelet features associated with the largest mutual information quotient $V_{F} / W_{c}$ were selected to construct the reduced feature set. This reduced feature set was then used to build other HMMs based on AICc. The AICc rewards goodness of fit based on the LL information, but also includes a penalty term that is proportional to the number of parameters $\left(K=3 Q M+Q^{2}\right)$ to reduce over-fitting.

$$
A I C c=2 \mathrm{~K}-2 L L+\frac{2 K(K+1)}{(n-K+1)}
$$

The size of the test set $(n)$ also plays a role in determining AICc if $n / K<40$. For each HMM topology ( $\mathrm{Q}$ and $\mathrm{M}$ ), an AICc value was computed. These AICc values were then rescaled with respect to the minimum AICc within the group such that the lowest AICc value was set to zero [31].

$$
\triangle A I C c=A I C c-\min (A I C c)
$$

The model with $\triangle \mathrm{AICc}<0.25$ while having the least number of model parameters (K) was then denoted as the optimal HMM $\left(\mathrm{HMM}_{\mathrm{AIC}}\right)$. 


\section{Statistical test and optimality index}

The HMM topologies were evaluated according to their abilities to detect non-seizure events, different stages in the tonic activities as well as chronic seizures. After the unsupervised training, the marginal posterior distribution $\gamma_{i}(t)$ for each state $i$ was computed by evaluating the HMMs on the test data. The detection of distinct initiation and termination of seizure dynamics as they evolved with time [42] was also considered in the form of early and late tonic spikes. An expert-guided state assignment procedure was used to identify non-ictal to ictal transitions using short time maximum Lyapunov exponent estimator derived from Rosenstein's algorithm [43]. The dynamics of chronic seizure period was first assigned to the most probable HMM state after training. Based on the state progression in the transition matrix, the tonic firing and non-ictal states were assigned in reverse order to HMM states before chronic activities; the postictal activity was assigned to the appropriate state forward-in-time to the HMM state after chronic activities.

Two types of spiking activities can be observed from the data set: tonic firing and chronic seizure activity. To evaluate the statistical robustness of the HMM, the sensitivity (TP) and specificity (TN) measures were determined. TP is defined by the percentage of correct chronic state detection within $30 \mathrm{~s}$ after the start of the chronic events, denoted as electrographical chronic seizure onset time (EcSOT) [44]. The percentage of correctly identified non-seizure/interictal activities at least $30 \mathrm{~s}$ prior to EcSOT is denoted as the TN. The ability of the HMM to detect chronic seizure onset early enough was determined. The time delay $(\Delta T)$ is defined as the time difference between the EcSOT and the approximated chronic seizure onset time (AcSOT) for each HMM topology. The AcSOT is defined as the time instance at which $\gamma_{i}(t)$ first identified the chronic seizure state within a detection horizon of $30 \mathrm{~s}$ around the EcSOT such that the EcSOT is located in the middle of a 1 min time window. Here, a positive $\Delta T$ would imply early chronic seizure detection while a negative $\Delta T$ would mean that the detection happened after the chronic seizure onset. Finally, the optimality index measure $(O)$ [32], used to evaluate the overall performance of the HMM, is defined as:

$$
O=\frac{T P+T N}{2}-\frac{\Delta T}{d}
$$

Here, the value $d$ is the chronic seizure duration for each SLE. A larger $O$ indicates a better overall performance for a given detector. These measures (TP, TN, $\Delta T$ and $O$ ) from the HMMs were also compared with other supervised wavelet artificial neural network (WANN) [6] built on identical features. Figure 2 provides a graphical illustration on the overall training and evaluation strategies.

\section{Results}

Comparison of the performance of $\mathrm{HMM}_{\mathrm{opt7D}}$ and $\mathrm{HMM}_{\mathrm{opt14D}}$ was done using a fivefold cross-validation technique. The model parameters of the HMMs were identified such that the best statistics for seizure detection in terms of optimality index $(O)$ can be produced in the validation data set.

After the unsupervised training and before the assignment of electrophysiological states to the model states, the LL for different combinations of $Q$ and $M$ values were 


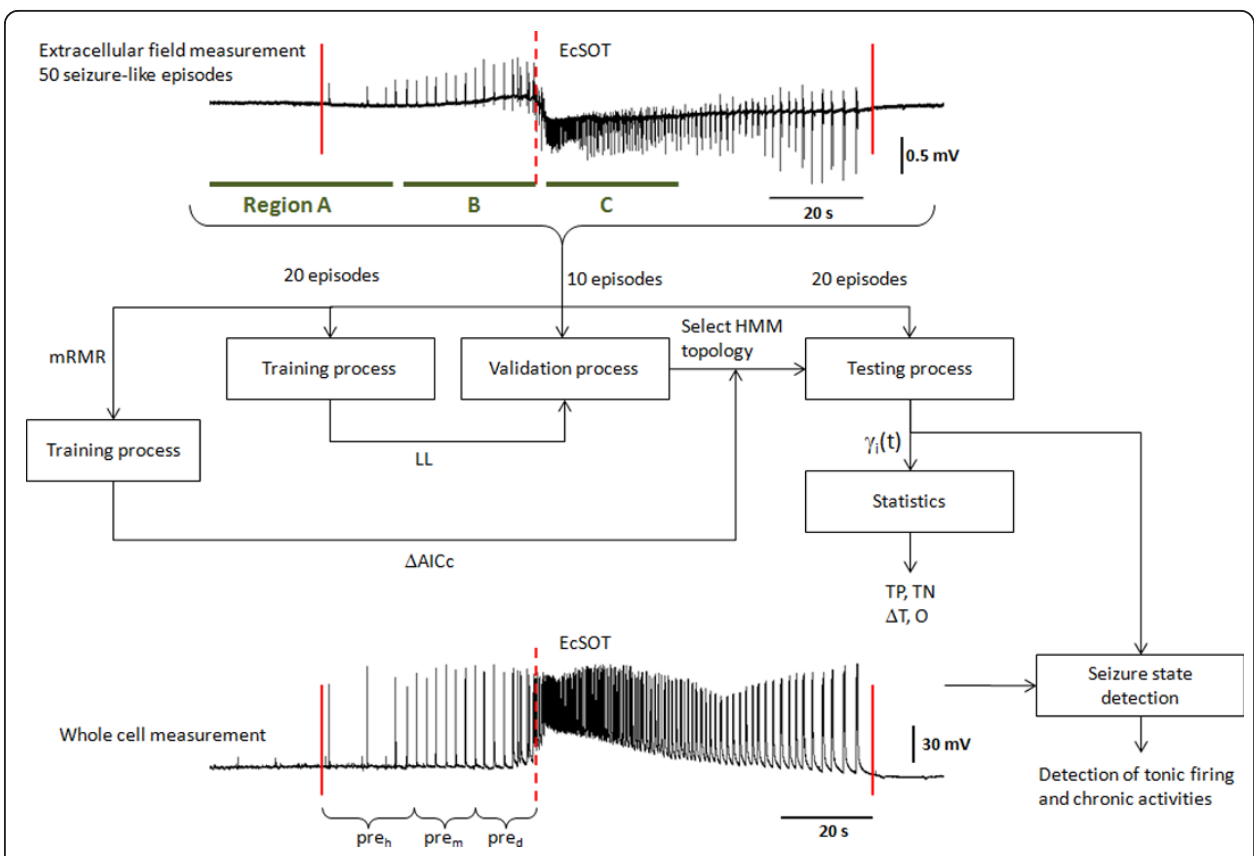

Figure 2 Graphical representation on the method of evaluating HMMs. On the top illustration, the LFP is illustrated. Maximum Lyapunov exponent analysis is used to determine the initiation and termination of seizure activities (marked by solid vertical lines). The seizure detection horizon is defined as a 1 min time window centered on the ECSOT (marked by dotted vertical line) and is denoted by regions B and C. After the initial training process (using $40 \%$ of the overall dataset) on the full 7-D and 14-D feature space, the performance of each HMM is evaluated using the validation set (20\% of the overall dataset) to determine the HMM $\mathrm{Opt}_{\mathrm{DD}}$ and $\mathrm{HMM}_{\mathrm{Opt} 14 \mathrm{D}}$ topology. Afterwards, feature reduction from mRMR analysis and AICC are used to find a suitable $H_{M M}$ AIC that balance the $L L$ against the number of model parameters. The statistical tests (TP and TN) as well as optimality index $(O)$ are then evaluated. On the bottom illustration, the intracellular activity before the EcSOT is partitioned based on its polarity characteristics into three states: hyperpolarizing $\left(\right.$ Pre $\left._{h}\right)$, depolarizing $\left(\right.$ Pre $\left._{d}\right)$ and a mixture of hyperpolarizing and depolarizing ( Pre $\left._{m}\right)$ activities [35]. This information is then compared to the $\gamma_{j}(\mathrm{t})$ associated with the tonic firing patterns in the HMM to evaluate the correlation between the multiple model states with the intracellular dynamics.

compared to give the initial estimate on the goodness of fit between the model and the recorded data. Not surprisingly, the LL on the training data would increase and gradually reach a plateau for large $\mathrm{Q}$ and $\mathrm{M}$ values. However, using a large number of states and clusters would cause over-fitting due to the curse of dimensionality, hence severely jeopardizing the generalization ability of the detector. It was also not surprising that at identical $\mathrm{Q}$ and $\mathrm{M}$ combination, the LL for the 14-D HMM using both $c$ and $\Delta c$ was always larger than the LL for the 7-D HMM using $c$ only (see Figure $3 \mathrm{a}$ and $3 \mathrm{~b}$ ). This implies that the feature space containing $\Delta c$ may more accurately represent the underlying dynamics of SLEs.

After assessing each HMM using the validation data, the topologies of the $\mathrm{HMM}_{\mathrm{opt7D}}$ and $\mathrm{HMM}_{\mathrm{opt14D}}$ were obtained with the expert-guided state assignment. For HMM with wavelet features $(c)$ only, the maximum sensitivity of $68.4 \pm 21.1 \%$ and specificity of $85.8 \pm 13.7 \%$ on the validation set was obtained for the HMM topology of $\mathrm{Q}=5$ and $\mathrm{M}=4$. The $a_{i j}$ of the $\mathrm{HMM}_{\mathrm{opt7D}}$ also exhibited a unidirectional sequence of state transitions (as shown in Figure 4a). The state $S_{5}$ was assigned as the chronic seizure state, $S_{5}$ was preceded by $S_{4}$ as the tonic firing state and $S_{2}$ as the interictal state. The $S_{1}$ and $S_{3}$ can then be consolidated to represent the postictal activity. An illustration of the LFP in the test set along with the posterior probabilities of the 


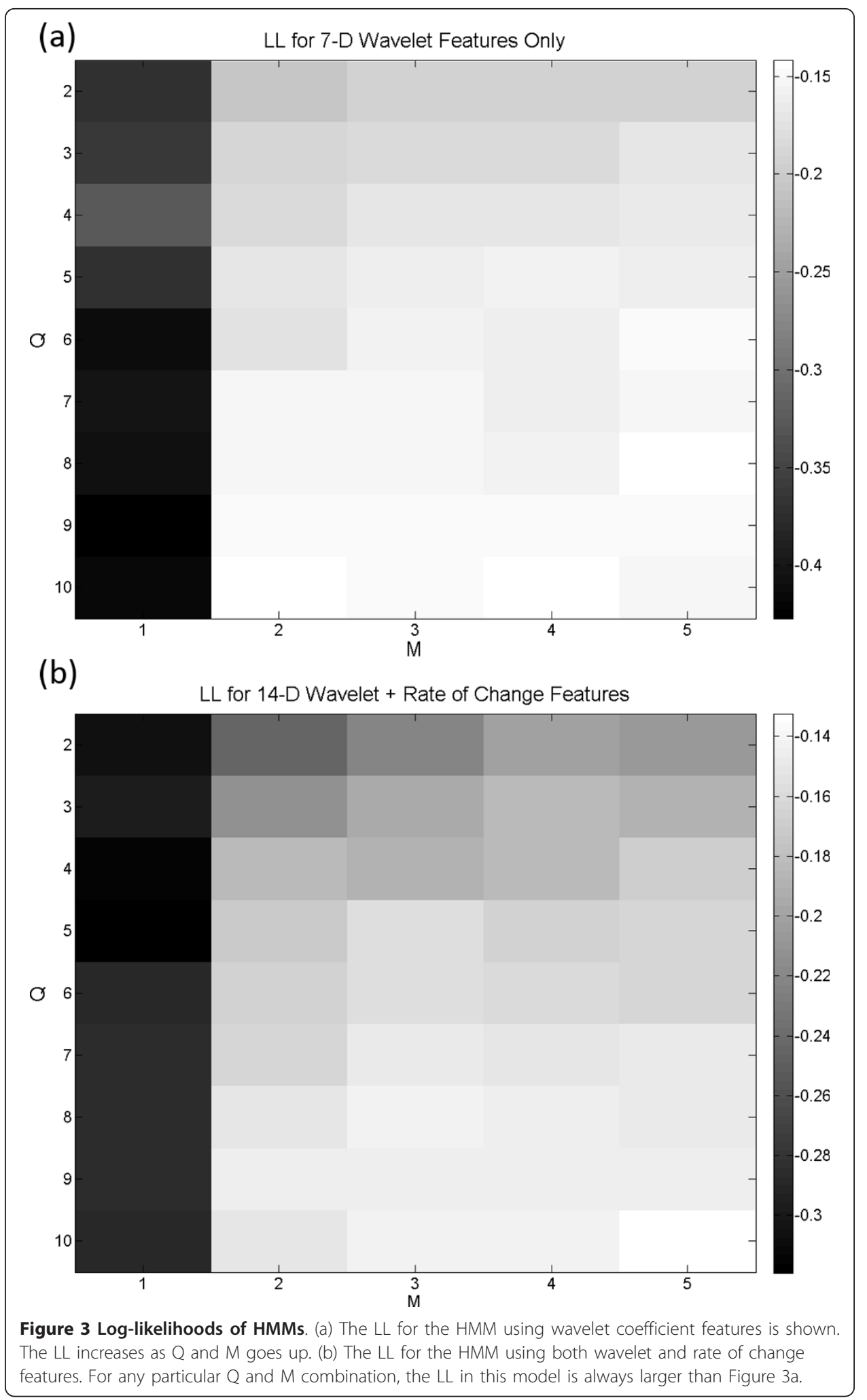




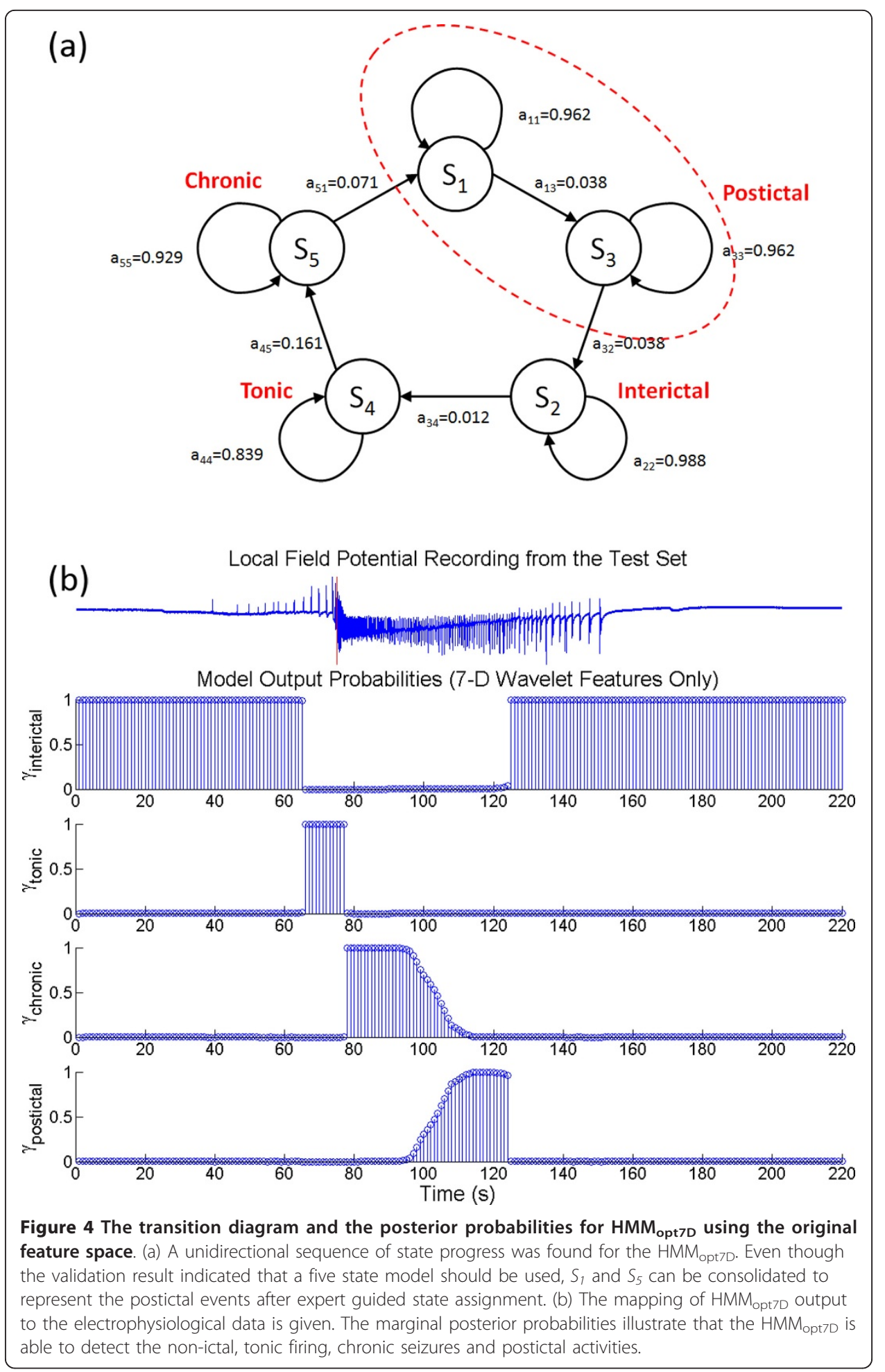

model states is shown in Figure $4 \mathrm{~b}$. The $\mathrm{HMM}_{\mathrm{opt14}} \mathrm{D}$ was found to contain $\mathrm{Q}=8$ and $M=3$, with the state transition diagram shown in Figure 5a. The application of $\mathrm{HMM}_{\mathrm{opt14D}}$ on the validation set gave the maximum sensitivity of $80.1 \pm 15.7 \%$ and maximum specificity of $95.3 \pm 4.4 \%$. The state $S_{6}$ can be assigned as chronic seizure, $S_{3}$ and $S_{8}$ can be assigned as the postictal and interictal respectively. The three 


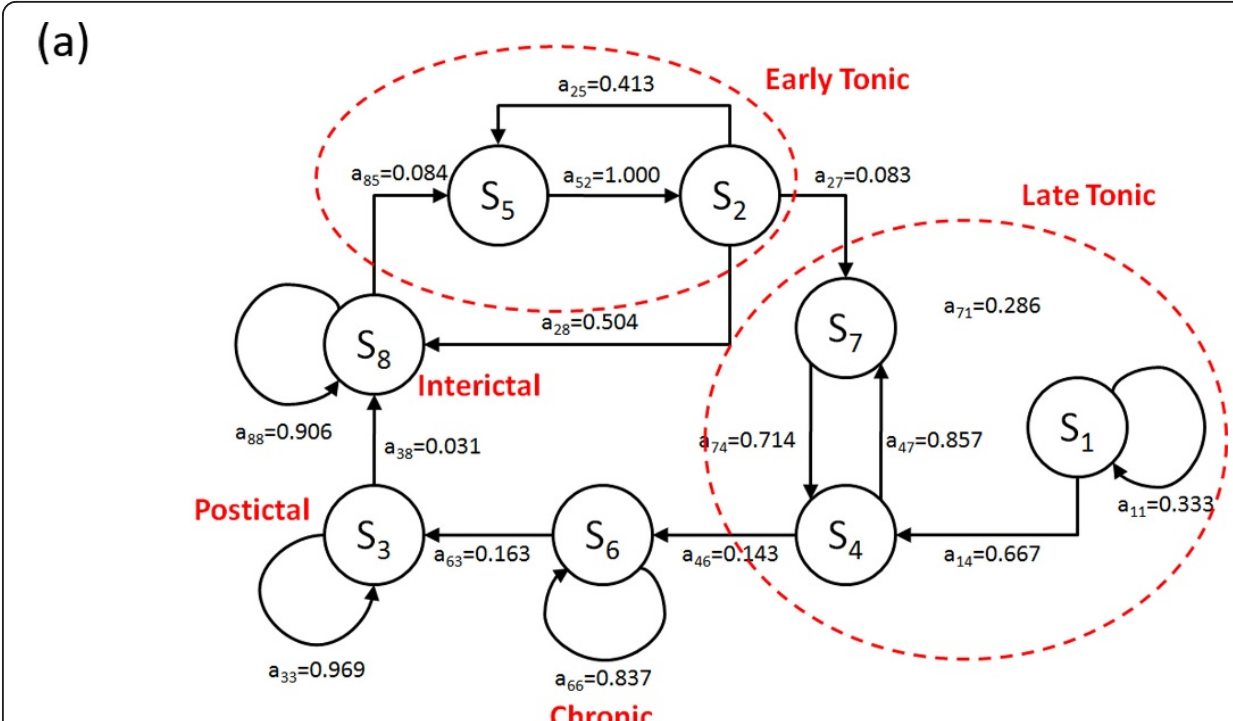

(b)

Local Field Potential Recording from the Test Set
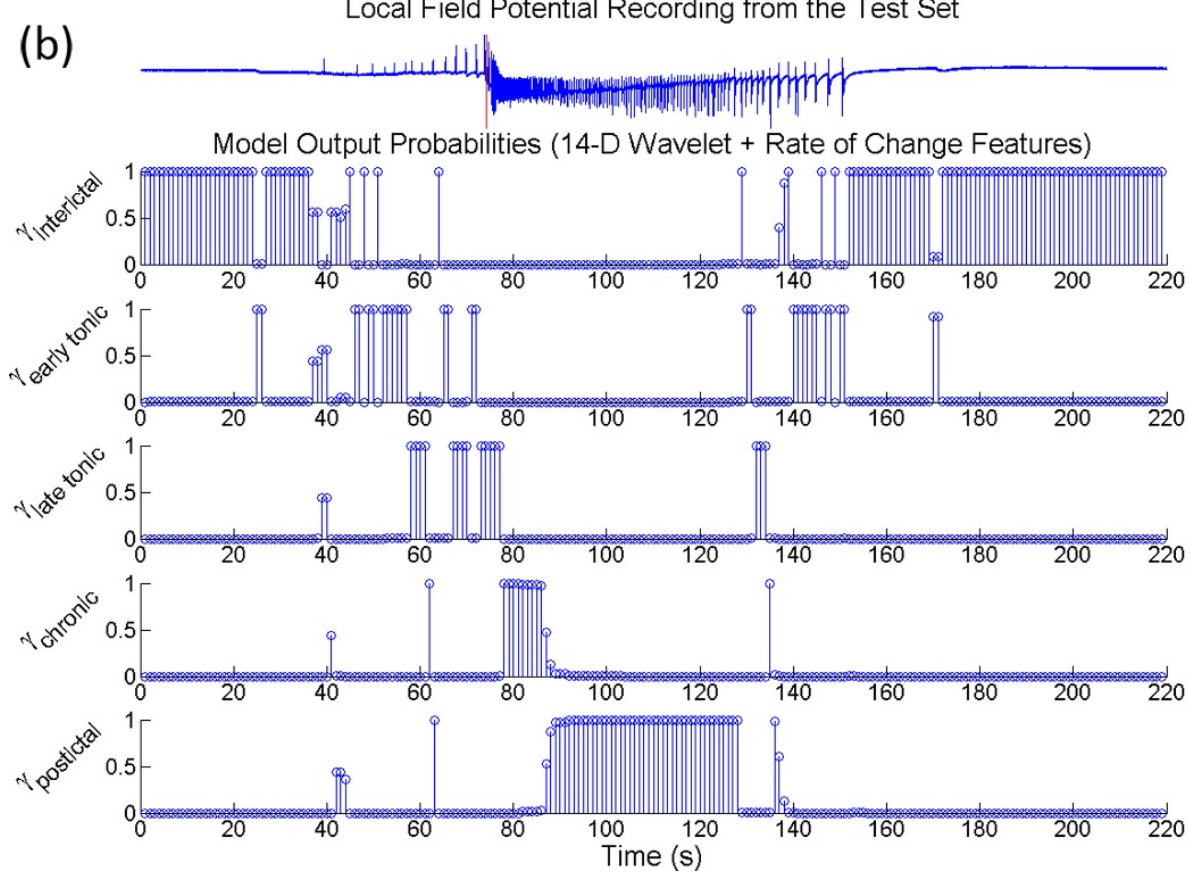

Figure $\mathbf{5}$ The transition diagram and the posterior probabilities for $\mathrm{HMM}_{\mathrm{opt} 14 \mathrm{D}}$ using the original feature space. (a) The state transition diagram for $\mathrm{HMM}_{\mathrm{Opt14D}}$ is given along with a sample LFP recording. For an eight state model, some of the states represent the same electrophysiological dynamics and hence can be consolidated. The HMM states $S_{1}, S_{4}$ and $S_{7}$ represent the late tonic activities. They are interconnected and are always followed by the chronic activity and preceded by early tonic firing states $S_{2}$ and $S_{5}$. The early tonic activity of this HMM is also allowed to return to interictal state without generating seizures. (b) The mapping of $\mathrm{HMM}_{\mathrm{opt} 14 \mathrm{D}}$ to the electrophysiological events is provided. Typically, this model is able to differentiate early and late tonic firing. For this particular example, the model output switches between interictal and early tonic state until about $46 \mathrm{~s}$.

interconnecting states of $S_{1}, S_{4}$, and $S_{7}$ before chronic state $S_{6}$ suggested that they can be assigned as the late tonic firing activities. The state transition followed a somewhat unidirectional evolution except for the interconnecting states of the early tonic state $\left(S_{2}\right.$ and $\left.S_{5}\right)$ back to the interictal state $\left(S_{8}\right)$, which appeared to suggest the possibility of having seizure permissive tonic firing not leading to chronic seizures. Figure $5 \mathrm{~b}$ gives a 
graphical illustration of the LFP along with the posterior probabilities of the model states using $\mathrm{HMM}_{\mathrm{opt14D}}$.

Next, the HMM approach was also compared against the supervised approach using identical wavelet coefficient feature set, in the form of a fully-supervised wavelet artificial neural network (WANN) seizure detection method designed by our group [6,7]. The training data for the WANN was created by first identifying the EcSOT, the data prior to the EcSOT was separated into $30 \mathrm{~s}$ intervals based on the assumption that there may be distinct changes in LFP leading to the EcSOT. Based on one-way ANOVA statistical analysis, the WANN and HMMopt7D did not show significant difference in their optimality index $(0.756 \pm 0.059$ and $0.665 \pm 0.260$, respectively).

When the wavelet rate of change information was included in the feature space, the $\mathrm{HMM}_{\mathrm{opt14D}}$ gave the best overall performance out of the three approaches, with $O=$ $0.915 \pm 0.302(\mathrm{p}<0.005)$. Table 3 summarizes the result of the chronic seizure detection for these three models.

The next question we asked was whether the state transitions of the HMM would hinder the performance of the state detection on seizure-free data as well as seizure permissive states that could translate back into interictal without generating seizures. Using $30 \mathrm{~min}$ of interictal bursts and non-seizure data that were not part of the training set, mean detection accuracies of $90.1 \%$ and $97.1 \%$ were achieved using $\mathrm{HMM}_{\text {opt7D }}$ and $\mathrm{HMM}_{\mathrm{opt14D}}$ respectively. Since the state transition of $\mathrm{HMM}_{\mathrm{opt7D}}$ is strictly unidirectional (Figure 4a), any missed false detection of the interictal state would trigger a cascade of errors, resulting in significantly lower accuracy in interictal state detection. $\mathrm{HMM}_{\mathrm{opt14D}}$ on the other hand allowed possible reversal in state transition back to interictal. In Figure 6, a sample LFP recording along with the posterior probabilities of the $\mathrm{HMM}_{\mathrm{opt14D}}$ is shown, illustrating that the model was able to classify the IB activities as non-ictal/interictal.

Another method for selecting the appropriate HMM topology was investigated by reducing the feature space dimension and the number of free parameters in the model. Minimum redundancy maximum relevance (mRMR) technique was used to select a subset of relevant wavelet features. It can alleviate the effect of over-fitting caused by the curse of dimensionality and improve the model's ability to generalize. In conjunction with the AICc, which helped balance the LL against the number of model parameters, suitable optimum labelled $\mathrm{HMM}_{\mathrm{AIC}}$ can be found. A summary of the mRMR analysis is shown in Table 4. The wavelet coefficients associated with the alpha (8 - 15 $\mathrm{Hz})$ and beta $(15-40 \mathrm{~Hz})$ bands had the largest mutual information quotient $\left(V_{F} / W_{c}\right.$ $=0.497$ and 0.436 respectively). The wavelet coefficients at these two frequency bands

Table 3 Performance measure for supervised and unsupervised seizure detection approach

\begin{tabular}{cccc}
\hline & WANN & HMM $_{\text {opt7D }}$ & HMM $_{\text {opt14D }}$ \\
\hline Sensitivity $(T P)$ & $73.1 \pm 3.7 \%$ & $69.8 \pm 20.3 \%$ & $86.7 \pm 27.2 \%$ \\
Specificity $(T N)$ & $91.7 \pm 4.4 \%$ & $88.1 \pm 20.9 \%$ & $98.6 \pm 7.7 \%$ \\
Detection delay $(\Delta T)$ & $3.95 \pm 3.38 \mathrm{~s}$ & $8.30 \pm 15.33 \mathrm{~s}$ & $-0.68 \pm 10.07 \mathrm{~s}$ \\
Optimality index $(\mathrm{O})$ & $0.756 \pm 0.059$ & $0.665 \pm 0.260$ & $0.915 \pm 0.302$ \\
\hline
\end{tabular}

Using identical wavelet coefficient features, the performance of the unsupervised HMM seizure detector $\left(\mathrm{HMM}_{\mathrm{Opt7D}}\right)$ did not show any statistically significant improvement over the supervised approach (WANN). However, when the rate of change of wavelet information was included in the feature space, the optimality index of $\mathrm{HMM}_{\mathrm{opt} 14 \mathrm{D}}$ was significantly better than both WANN and $\mathrm{HMM}_{\text {opt7D. }}$ 


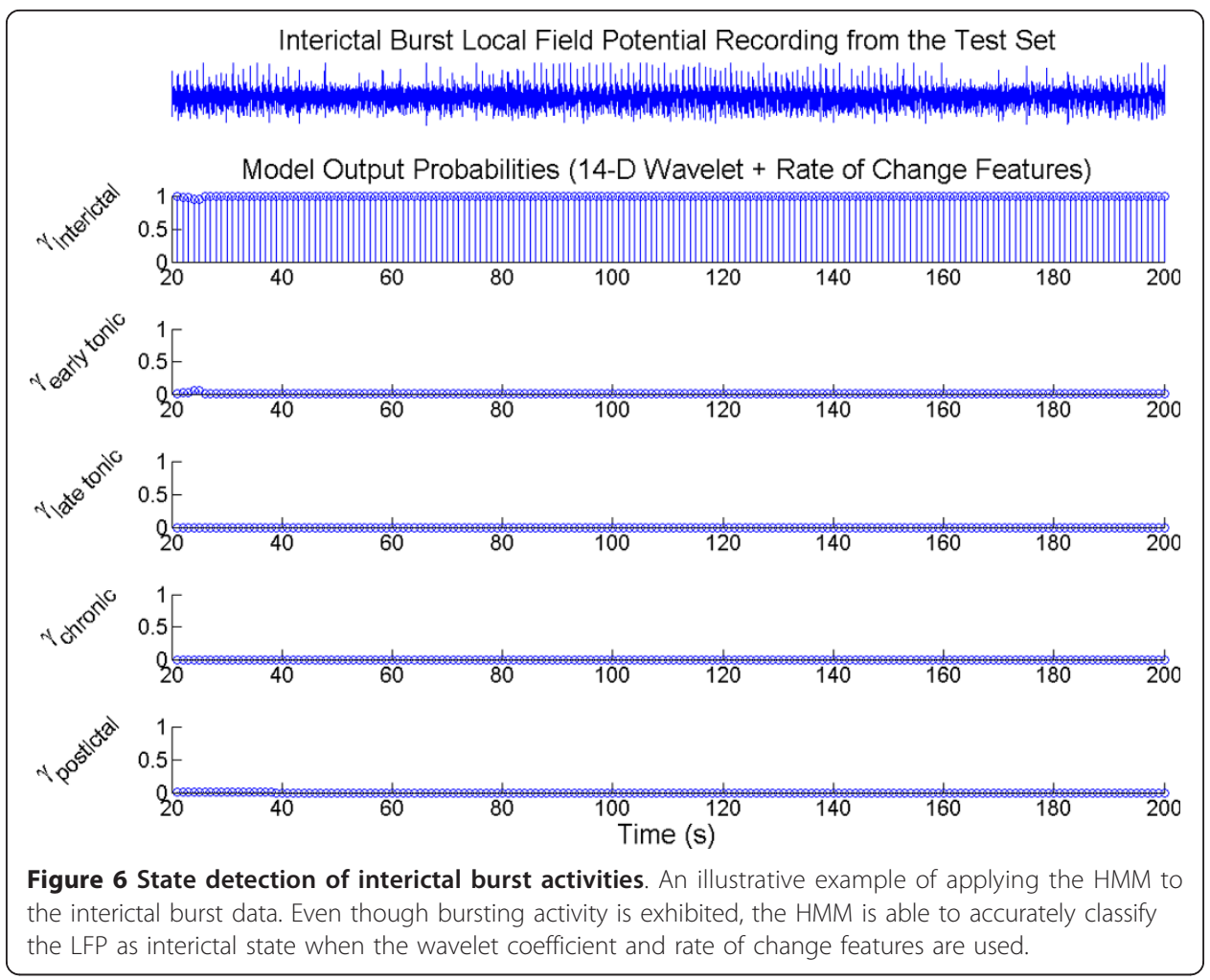

then constituted the reduced features space. For the feature space consisted of the wavelet coefficients $(c)$ only, the simplest 2-D HMM topology with $\triangle \mathrm{AICc}<0.25$ was found at $Q=3, M=2$ (Figure 7a). The state transition diagram of the corresponding HMM is shown in Figure 8a. It contained three bidirectionally connected states. When the posterior probabilities of this model were matched against the LFP after assigning the model states (Figure 8b), no distinction between the tonic firing and the postictal events was found. There were also cases in which the state transitions jumped from interictal to chronic seizure directly and back. ON average, the performance of this $\mathrm{HMM}$ was slightly better than the $\mathrm{HMM}_{\text {opt7D }}$, with $\mathrm{TP}=80.9 \pm 34.4 \%, \mathrm{TN}=94.8 \pm$ $15.6 \%, \Delta T=3.79 \pm 8.67 \mathrm{~s}$ and $O=0.813 \pm 0.247$, even though student $\mathrm{T}$-test analysis did not reveal any statistically significant improvement. When the wavelet rate of change information $(\Delta c)$ was added as feature, a 4-D reduced feature space was created. The simplest topology with $\triangle \mathrm{AICc}<0.25$ for this feature set was found at $\mathrm{Q}=5$

Table 4 Feature selection using mRMR method

\begin{tabular}{ccc}
\hline Order & $\mathbf{V}_{\mathbf{F}} / \mathbf{W}_{\mathbf{c}}$ & Frequency Range $(\mathbf{H z})$ \\
\hline 1 & 0.497 & $8-15$ \\
2 & 0.436 & $15-40$ \\
3 & 0.400 & $100-250$ \\
4 & 0.331 & $4-8$ \\
5 & 0.308 & $40-100$ \\
6 & 0.308 & $250-400$ \\
7 & 0.266 & $<4$ \\
\hline
\end{tabular}

The frequency bands associated with the two highest mutual information quotients $\left(V_{F} / W_{c}\right)$ were used to create the reduced feature space. In our analysis of chronic seizures versus non-seizure activities, the alpha $(8-15 \mathrm{~Hz})$ and beta (15 - $40 \mathrm{~Hz}$ ) activities were found to be the most relevant for seizure detection. 
(a) $\Delta \mathrm{AlC}_{\mathrm{c}}$ for 2-D Wavelet Features Only

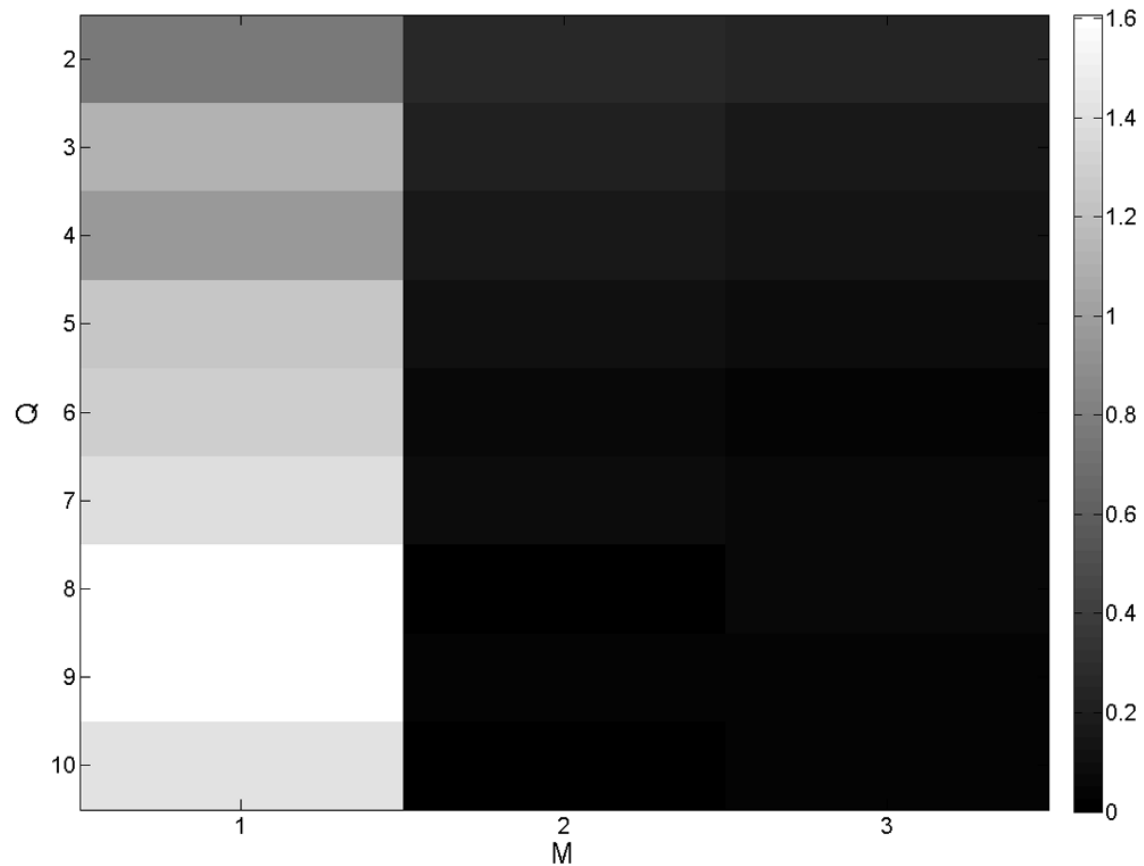

(b) $\Delta \mathrm{AlC}_{\mathrm{c}}$ for 4-D Wavelet + Rate of Change Features

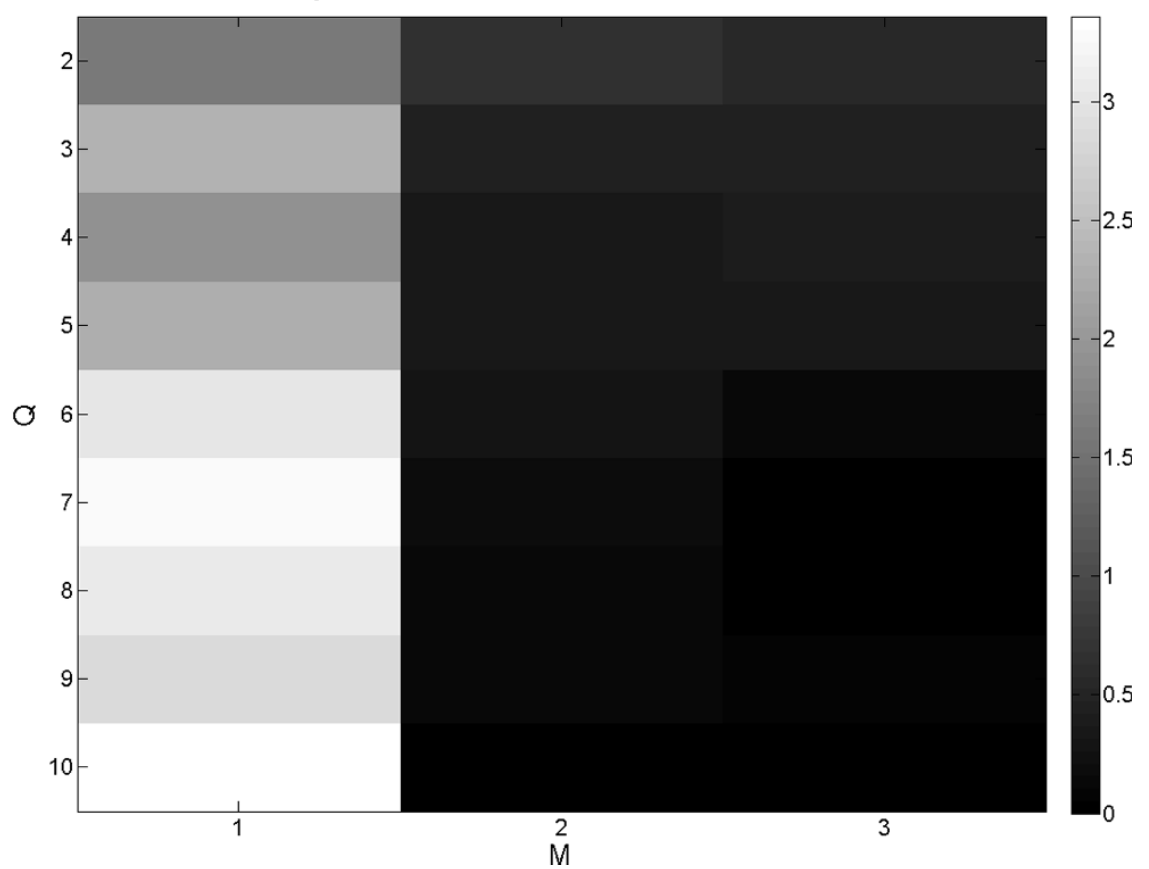

Figure 7 The rescaled AICC values are plotted with respect to the number of HMM states and Gaussian mixtures. (a) The simplest model with $\triangle \mathrm{AICC}<0.25$ is at $\mathrm{Q}=3$ and $\mathrm{M}=2$. In this model, the reduced feature space consists of 2-D wavelet coefficients. (b) With the 4-D wavelet and rate of change features established using mRMR, the simplest model with $\triangle \mathrm{AIC} c<0.25$ is found at $Q=5, M=3$. 
(a)

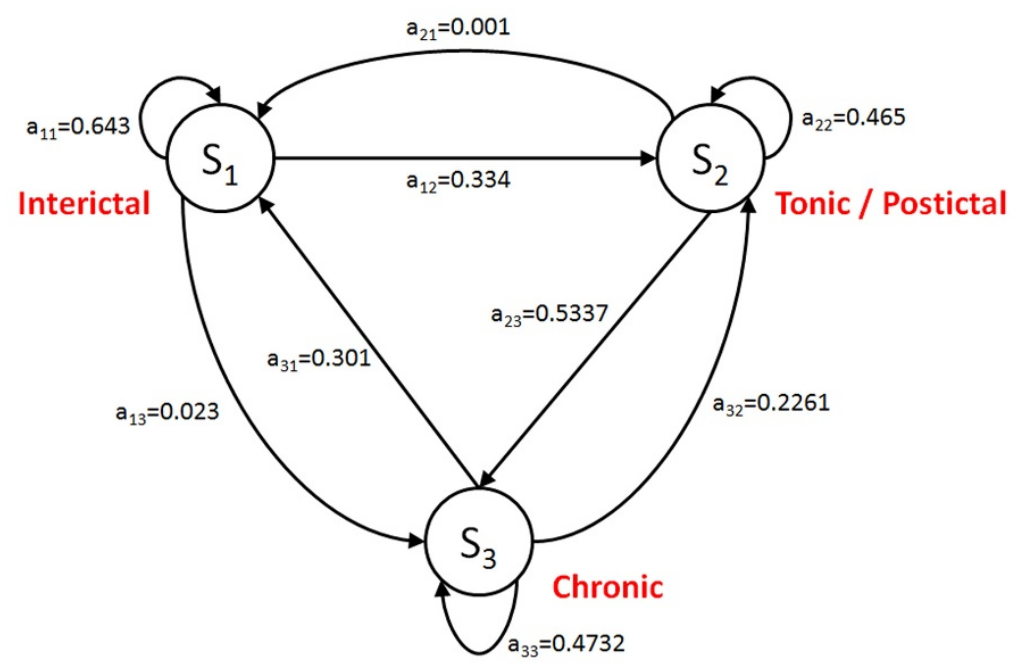

(b)

Local Field Potential Recording from the Test Set
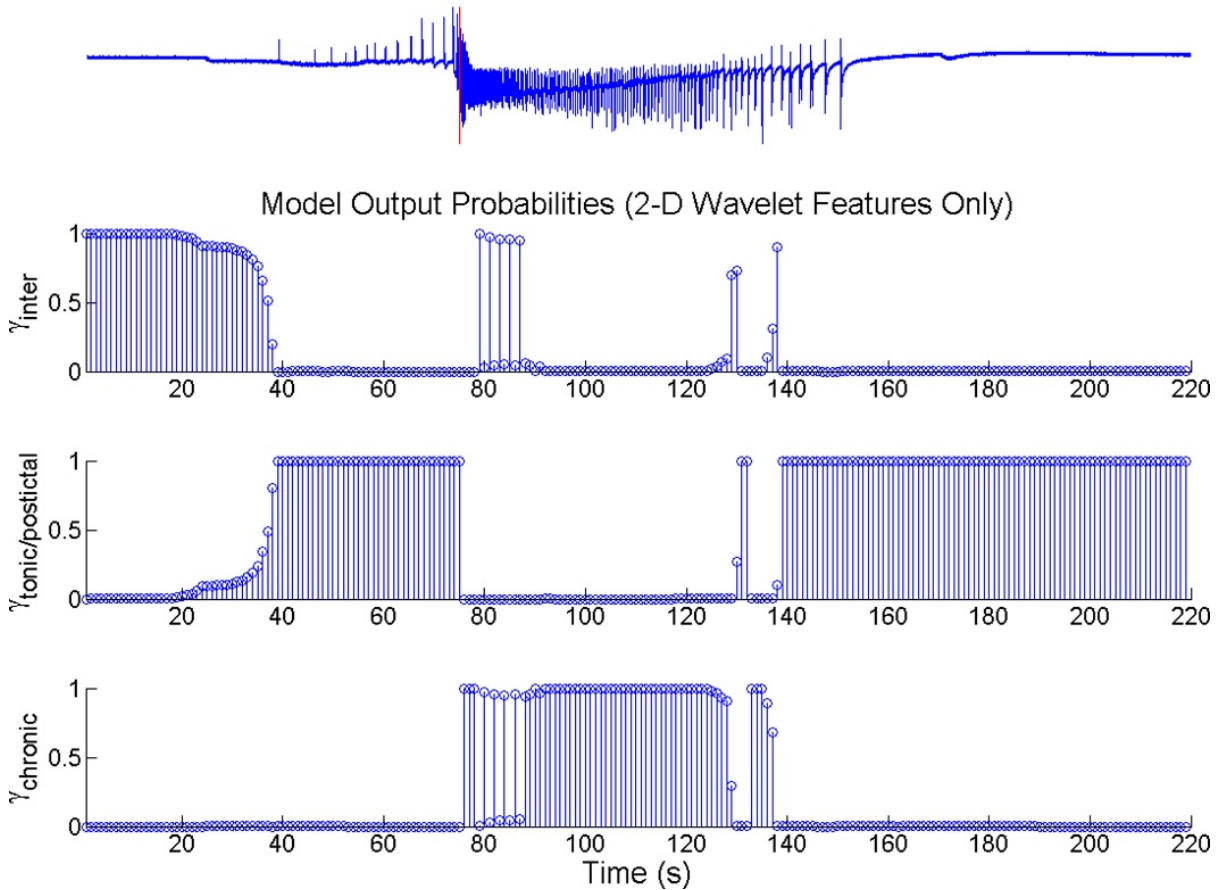

Figure 8 The transition diagrams and the posterior probabilities of the reduced feature space after mRMR and AICc. (a) A bidirectional sequence of state progress is found where the HMM is not able to distinguish between tonic firing and postictal activities. (b) The mapping of the HMM output to the experimental data is shown. The tonic firing phase prior to the chronic seizure and the postictal activity are represented by the same model state. Because the state transition probability from the tonic/postictal state is extremely small (0.001), it would take much longer for the interictal activity right after chronic seizure to be classified correctly.

and $M=3$ (Figure $7 \mathrm{~b}$ ). Figure 9a shows the state transition diagram for this HMM (called $\mathrm{HMM}_{\mathrm{AIC}}$ ). Similar to the $\mathrm{HMM}_{\mathrm{opt14D}}$, the state progression was mainly unidirectional with a non-zero state transition probability from the early tonic state back to the interictal state. This suggests that it is possible to have seizure permissive early tonic activity that can be reverted back to the interictal state. The marginal posterior 


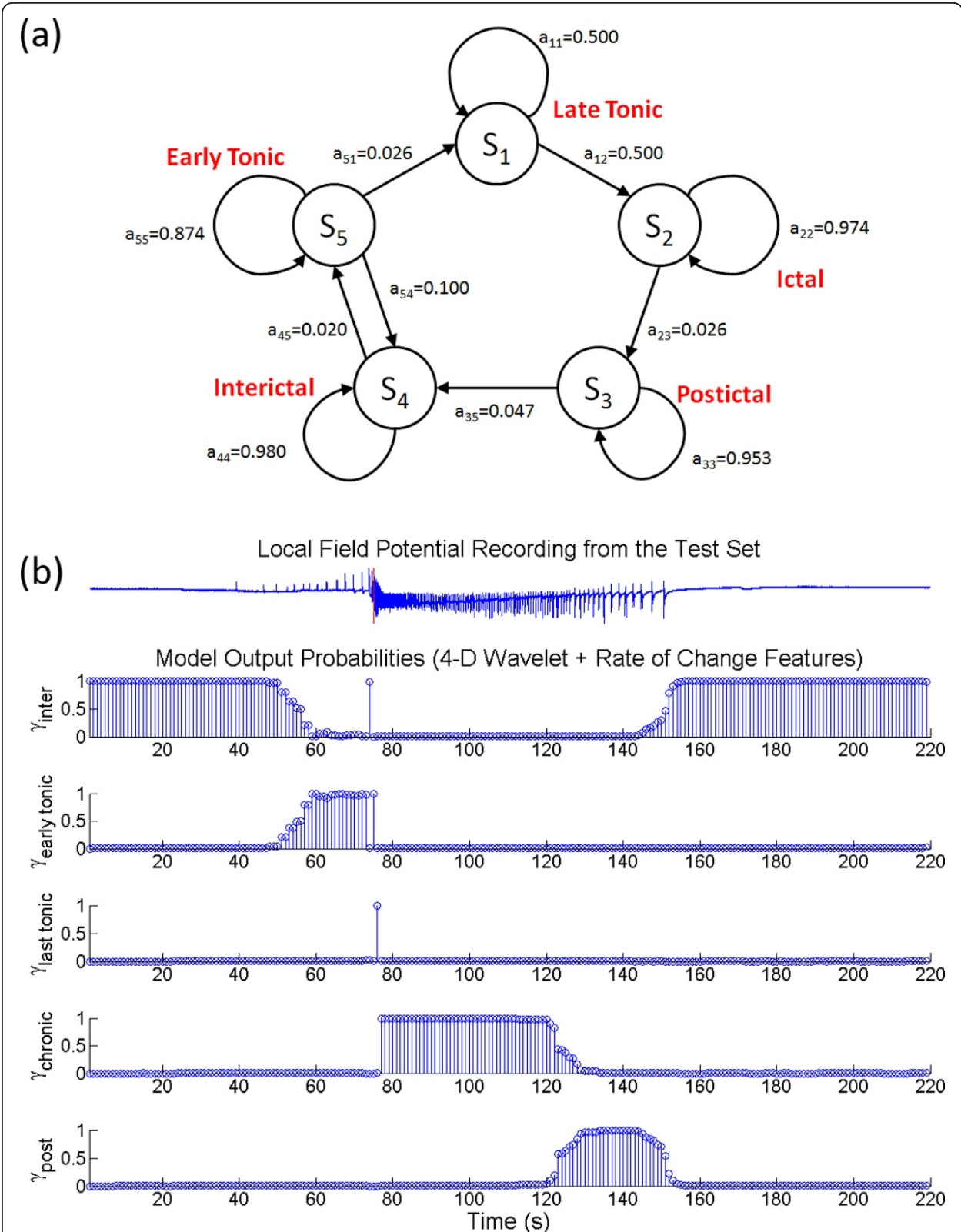

Figure 9 The transition diagrams and the posterior probabilities of the reduced feature space after mRMR and AICc. (a) The state transition diagram for HMM AIC $_{\text {is }}$ given. Similar to the HMM $\mathrm{Hopt}_{14 \mathrm{D}}$, the some early tonic activity permissive to seizures is allowed to return to the interictal state. Between the late tonic firing and postictal state, the transition follows a unidirectional progression. (b) The mapping of $\mathrm{HMM}_{\mathrm{AIC}}$ states to the electrophysiological LFP is shown. In this illustrative example, the marginal probabilities of the model states follow a progression from non-ictal, tonic firing to chronic seizures and postictal state, before returning to non-ictal. The late tonic firing state in this model is typically shorter than the model from Figure $5 b$ because of the significantly smaller transition probability back to itself.

probability $\gamma_{i}(t)$ for each state $i$ of $\mathrm{HMM}_{\mathrm{AIC}}$ is plotted against a sample test data in Figure 9b. Even though no significant improvement over $\mathrm{HMM}_{\mathrm{opt14D}}$ was revealed using the student $\mathrm{T}$-test, the $\mathrm{HMM}_{\mathrm{AIC}}$ gives the best overall performance out of all the HMMs created in this study with $\mathrm{TP}=95.7 \pm 14.0 \%, \mathrm{TN}=98.9 \pm 6.5 \%, \Delta \mathrm{T}=-2.03 \pm$ $7.10 \mathrm{~s}$ and $O=0.995 \pm 0.129$. A summary of the performance measures for the $\mathrm{HMM}_{\mathrm{AIC}}$ using mRMR and $\triangle \mathrm{AICc}$ is presented in Table 5. 
Finally, we evaluated the correlations between the model tonic firing state from the $\mathrm{HMM}_{\mathrm{AIC}}$ with the phasic inhibition or excitation in the intracellular activities [35]. It has been suggested that the intracellular whole-cell recordings exhibited a switch from a dominant phasic inhibition ( pre $_{h}$ ) to a dominant phasic excitation $\left(\right.$ pre $\left._{\mathrm{d}}\right)$ mode in the state transition leading to the chronic seizure onset [35]. An intermediate state ( pre $_{m}$ ) was also reported to compose of a mixture of $\mathrm{pre}_{\mathrm{h}}$ and $\mathrm{pre}_{\mathrm{d}}$ mode. None of the HMM created in this study was able to detect early $\mathrm{pre}_{\mathrm{h}}$ mode using the LFP data. In the $\mathrm{HMM}_{\mathrm{opt14D}}$, the states $S_{2}$ and $S_{5}$ (Figure 5a) can be considered as a combination of late pre $_{\mathrm{h}}$ and $\mathrm{pre}_{\mathrm{m}}$. Figure 10a summarizes the temporal relationship between the HMM-identified early and late tonic firing activities in the LFP with the identified $\mathrm{pre}_{\mathrm{m}}$ and $\mathrm{pre}_{\mathrm{d}}$ modes in the whole-cell recording. Most of the late tonic firing activities identified by the model started earlier than the pre ${ }_{\mathrm{d}}$ intracellular activities. Out of the 20 test cases, $85.9 \%$ of the pre $\mathrm{d}_{\mathrm{d}}$ activity was identified as the late tonic firing phase. A paired T-test did not indicate a statistically significant difference between the start of the whole-cell pre $\mathrm{d}_{\mathrm{d}}$ activity and the onset of the late tonic HMM state $(\mathrm{p}>0.49)$, as illustrated in Figure 10b.

\section{Discussion}

The administration of a therapeutic intervention, such as electrical stimulation, may be effective in preventing seizures before and during the early stages of the seizure onset [45]. The success of a real-time closed-loop seizure prevention method depends on the time available between the early seizure detection and the manifestation of ictal onset. Many early seizure detection algorithms have been proposed $[9,15,23,32,46-50]$. The application of HMM for seizure dynamics detection was inspired by the relatively poor objective criteria for identifying the precise period of preictal interval in supervised learning. Here, an unsupervised training strategy with expert guided state assignment of HMM is proposed where the best rule to represent the wavelet features of seizure progression can be identified. One of the major criteria for selecting an appropriate seizure detection algorithm for a real-time seizure intervention system is the available time for the detection of the impending ictal onset. In this paper, HMMs were created utilizing different feature vectors to characterize the dynamics of SLEs. A motivation behind this work is to determine whether an unsupervised method can produce an accurate seizure detector with a high optimality index. Furthermore, feature selection based on the mRMR criteria and topology selection based on $\triangle \mathrm{AICc}$ were implemented to evaluate the ability of the HMM to detect multi-stage dynamics leading to

Table 5 A summary of HMM performance measures

\begin{tabular}{ccccc}
\hline Features & \multicolumn{2}{c}{ Wavelet Coefficients Only } & \multicolumn{2}{c}{ Wavelet Coefficients + Rate of Change } \\
\hline & Validation Set & mRMR and $\Delta$ AICc & Validation Set & mRMR and $\Delta$ AICc \\
& 7-D $(\mathbf{Q}=\mathbf{5}, \mathbf{M}=\mathbf{4})$ & 2-D $(\mathbf{Q}=\mathbf{3}, \mathbf{M}=\mathbf{2})$ & $\mathbf{1 4 - D}(\mathbf{Q}=\mathbf{8}, \mathbf{M}=\mathbf{3})$ & 4-D $(\mathbf{Q}=\mathbf{5}, \mathbf{M}=\mathbf{3})$ \\
\hline Sensitivity $(\mathrm{TP})$ & $69.8 \pm 20.3 \%$ & $80.9 \pm 34.4 \%$ & $86.7 \pm 27.2 \%$ & $95.7 \pm 14.0 \%$ \\
Specificity $(\mathrm{TN})$ & $88.1 \pm 20.9 \%$ & $94.8 \pm 15.6 \%$ & $98.6 \pm 7.7 \%$ & $98.9 \pm 6.5 \%$ \\
Detection delay $(\Delta \mathrm{T})$ & $8.30 \pm 15.33 \mathrm{~s}$ & $3.79 \pm 8.67 \mathrm{~s}$ & $-0.68 \pm 10.07 \mathrm{~s}$ & $-2.03 \pm 7.10 \mathrm{~s}$ \\
Optimality index $(\mathrm{O})$ & $0.665 \pm 0.260$ & $0.813 \pm 0.247$ & $0.915 \pm 0.302$ & $0.995 \pm 0.129$ \\
\hline
\end{tabular}

The optimal HMM model topologies were determined using two methods. The first method involved the use of validation data set consisting of 10 SLEs to find the HMM topology that gave the highest optimality index. The second method used the mRMR feature selection method and AICc to reduce the feature space dimension and the complexity of the HMM. The HMM obtained using mRMR and AICc has the highest optimality index. 


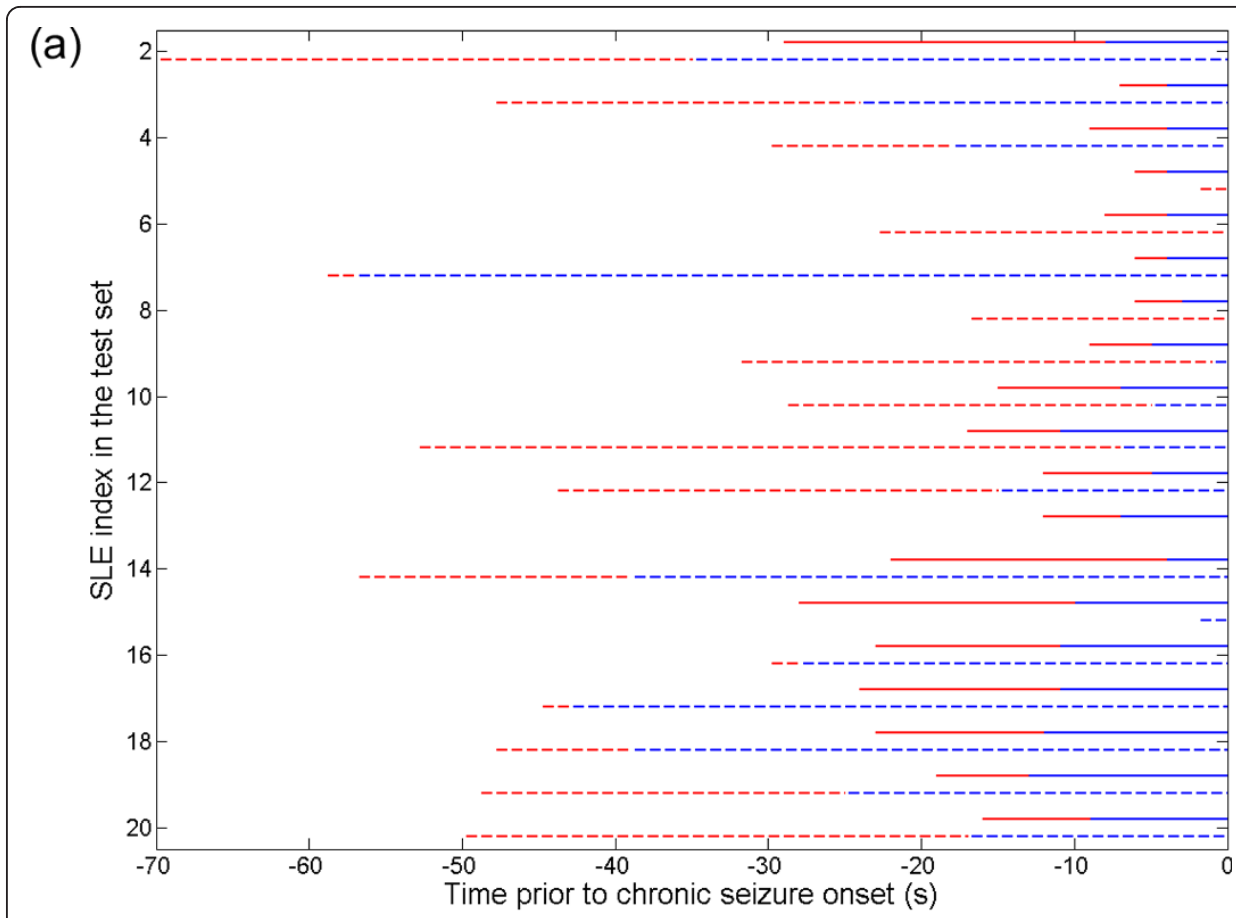

(b)

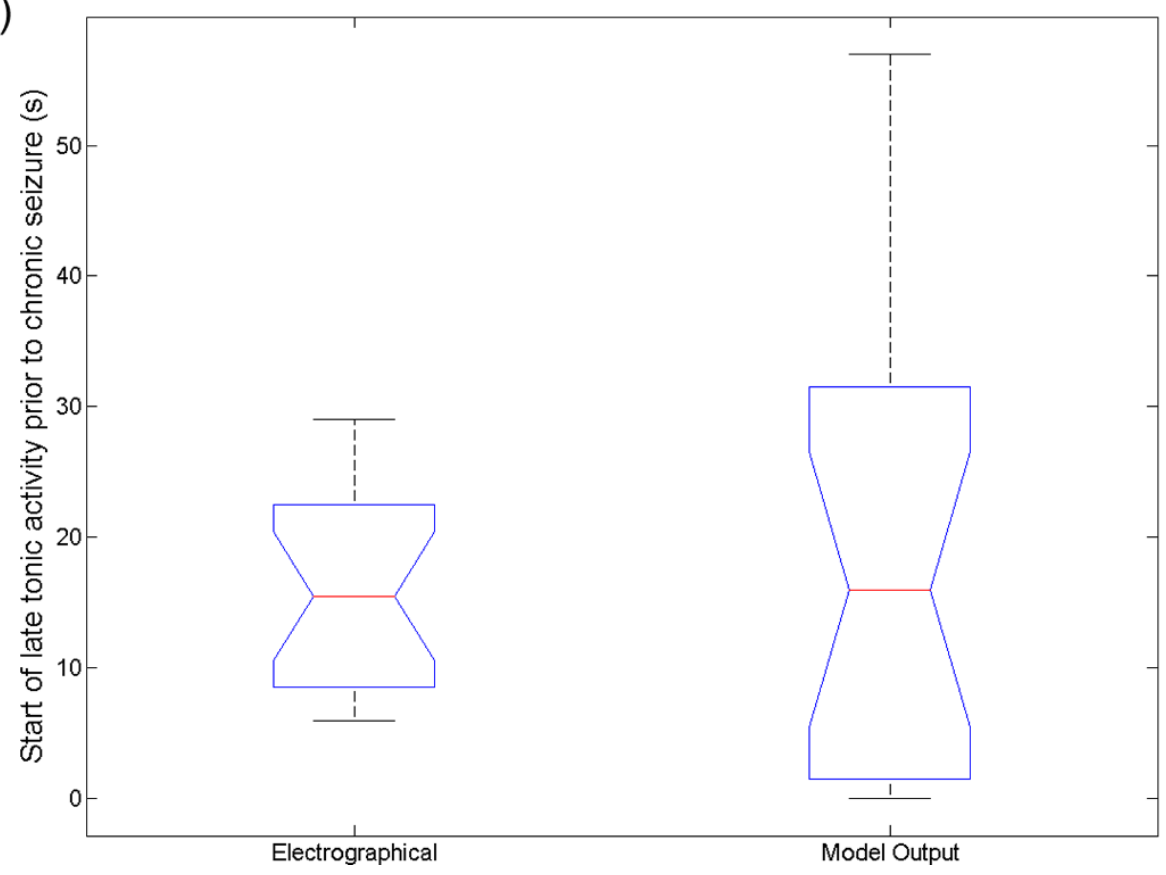

Figure 10 The relationship between the HMM identified late tonic states and the intracellular modes. (a) The temporal relationship between the intracellular modes before chronic seizures and the HMM late tonic firing states for each SLE in the test set is shown. The intracellular modes are shown in solid color (red for pre $\mathrm{m}_{\mathrm{m}}$ and blue for pre $_{\mathrm{d}}$ ). The identified states by the HMM are shown as dashed lines (red for early tonic and blue for late tonic states). (b) The boxplot summarizes the relationship between the

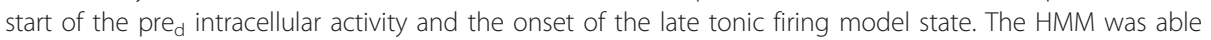
to identify $85.9 \%$ of the pre $\mathrm{d}_{\mathrm{d}}$ activity as the late tonic firing state. The mean starting times for the electrographical pre $e_{d}$ activity and the model late tonic state were $16.25 \mathrm{~s}$ and $16.77 \mathrm{~s}$ prior to chronic seizure onset, respectively. Using pair-wise T-test analysis, no statistically significant difference was found between the two $(p>0.49)$. 
chronic seizure onset by measuring the sensitivity, specificity, detection delay and the optimality index.

The unsupervised learning strategy of the HMM involves the estimation of the model parameters through the maximization of the LL function. The training data consists of the wavelet-based features from 20 SLEs. Unlike other recent approaches that assumed a fixed number of states (such as interictal, preictal and ictal) in the model [28], the total number of hidden states in the HMM in this study was allowed to vary in an unsupervised manner. It is logical to expect that if more states are allowed, the better the model would match the observations. However, increasing the possible number of hidden states would lead to over-fitting, hence reducing its ability to generalize. To alleviate this problem, we need to find out the optimal HMM topology defined by the number of states $(\mathrm{Q})$ and the number clusters in the feature space (M) that would be able to generalize well.

The first method we tried was to set aside a portion of the data for validation purpose. The objective was to get the $\mathrm{Q}$ and $\mathrm{M}$ combination that would give the highest optimality index in the validation set. The optimal HMM $\left(\mathrm{HMM}_{\mathrm{opt7D}}\right)$ created using only wavelet coefficient features had five states $(\mathrm{Q}=5)$ with each feature space modeled by four clusters $(M=4)$. If the rate of change of the wavelet coefficients was also included in the feature space, the optimal HMM $\left(\mathrm{HMM}_{\mathrm{opt14}}\right)$ would consist of eight states $(\mathrm{Q}=8)$ with the feature space modeled using three clusters $(M=3)$. The $H M M_{\mathrm{opt14D}}$ is superior to the $\mathrm{HMM}_{\mathrm{opt7D}}$ not only because it has a higher optimality index $(0.915 \pm 0.129$, compared to $0.665 \pm 0.260$ ), but also because it is more robust in identifying multiple distinct dynamics between non-ictal and ictal events. The detection of early and late tonic firing activities prior to the chronic onset in vitro also became possible. While the training process of the HMM was not constrained to follow a unidirectional state transition, the state transitions leading to the ictal onset often possessed some unidirectional progress. Once the $\mathrm{HMM}_{\mathrm{opt7D}}$ output moved away from the interictal state, it must go through the whole seizure progression before returning to interictal. This would then increase the number of false detection in $\mathrm{HMM}_{\mathrm{opt7D}}$, as indicated by the results shown in Table 3. On the other hand, some early tonic firing state in the $\mathrm{HMM}_{\mathrm{opt14D}}$ was allowed to revert back to interictal directly. However, once it had advanced past the early tonic state, it could not revert back to non-ictal activities. The HMM $\mathrm{opt14D}_{\mathrm{D}}$ was successfully tested on non-seizure or interictal burst data, demonstrating a mean accuracy of over 97\%. Next, the unsupervised HMM and supervised WANN approaches [6] were compared. The two methods differ in the way that the parameters are obtained. The parameters of the HMM were updated in an iterative manner until no significant improvement in LL was achieved. The supervised WANN approach, on the other hand, required that the human user separated the data into different training groups. The WANN parameters were updated iteratively based on the partial derivative of error with respect to the weights. By definition, the performance of any supervised learning algorithm can be no better than the initial separation of seizure states by the human expert's "Gold Standard". Since the human user has access to the entire observation before marking the chronic onsets, retrospective bias exists in any supervised learning algorithm [28]. This retrospective bias can be eliminated with an unsupervised algorithm such as HMM. The HMM implementation is superior to the WANN because it can distinguish between the early and late tonic firing without having to define them before training. 
The second method to reduce the complexity of the HMM based on mRMR feature selection criteria and $\triangle \mathrm{AICc}$ topology selection was evaluated. The mRMR analysis showed that activity in the alpha $(8-15 \mathrm{~Hz})$ and beta $(15-40 \mathrm{~Hz})$ range has the largest mutual information quotient $\left(V_{F} / W_{c}\right)$ of 0.497 and 0.436 respectively. This result is consistent with the existing literature showing that the alpha and beta frequency bands exhibit considerable difference in the signal complexity between healthy subjects, epileptic subjects during a seizure-free interval, and during seizure [8]. Two new feature spaces were constructed: One consisted of 2-D wavelet coefficients and one consisted of 4-D wavelet coefficient with rate of change information. Using these new feature spaces, HMMs with different topologies were created using unsupervised learning. Since having large number of $\mathrm{Q}$ and $\mathrm{M}$ often lead to the curse of dimensionality resulting in over-fitting, AICc was used to select the appropriate model topology by balancing the goodness of fit with the number of parameters used. We found that many $\mathrm{Q}$ and $\mathrm{M}$ combinations were able to achieve similar levels of AICc; the optimal HMM was selected as the model with the smallest number of parameters while maintaining a difference of $<0.25$ from the minimum AICc. The HMM of 2-D reduced features consists of three states $(\mathrm{Q}=3)$ with two clusters $(M=2)$. One of these three states reflects the dynamics of both tonic firing and postictal activities (Figure 8a). Because of the small transition probability $\left(a_{i j}=0.001\right)$ from the tonic/postictal back to interictal state, it typically would take a much longer time for the model to return to the proper interictal state after chronic seizure. This has been the source for most of the misclassification in the non-ictal activity using this model. The HMM model of 4$D$ reduced features consists of five states $(Q=5)$ with three clusters $(M=3)$. Again, by including the wavelet rate of change information, this model gave the best overall performance with $O=0.995 \pm 0.129$, even though the use of AICc does not warrant an improvement in seizure detection performance. The AICc simply offers a much simpler alternative to selecting the optimal HMM topology. We were able to more easily and more quickly create a HMM seizure detector with fewer number of model states and feature clusters with similar performance level.

The interpretation of early and late tonic firing state detected using $\mathrm{HMM}_{\mathrm{AIC}}$ in terms of the underlying intracellular whole-cell measurement was investigated. When the model posterior probabilities were plotted against the corresponding intracellular whole-cell recording, we found a significant overlap ( $>85 \%)$ between the late tonic state and the intracellular pre $_{\mathrm{d}}$ activity. It was also observed that the early hyperpolarizing activity $\left(\mathrm{pre}_{\mathrm{h}}\right.$ ) could not be distinguished from the interictal activities.

The application of HMM is based on the assumption that state transitions in seizure generation follow first-order Markov processes. Depending on the sampling period of the signal and the available history of HMM output, it is likely that neurodynamic would depend on more than one previous sample in time. This work can be extended to incorporate additional states in the past for the estimation of state transitions, which can be achieved through modification of the conditional state transition probability equation such as the hidden semi-Markov model [51] where state transition depends on the lapsed time since entering the current state. This model is also appropriate because the underlying process of seizure generation may not have a geometrically distributed duration. Another possible improvement is to include other types of features such as multi-site coherence in the feature space, since the manifestation of 
epilepsy typically involved progressive global entrainment. Preliminary analysis on the analysis of clinical seizure data from intracranial EEG measurement using HMM also showed promising results [52].

\section{Conclusions}

Based on the analysis of optimality index, the implementation of hidden Markov model as a seizure dynamics detector offers significant improvement over existing approaches based on human visual classification and supervised connectionist perspectives. The subjectivity involved in partitioning the observed data into target states prior to training is eliminated. This model is able to estimate the parameters needed to best fit the observed data depending on its specified topology. Once the unsupervised training is completed, the assignment of the model state to the electrophysiology data is guided by a human expert. To alleviate the curse of dimensionality, feature selection based on minimum redundancy maximum relevance and topology selection based on Akaike information criteria are implemented without jeopardizing the sensitivity, specificity and early detection time of the final model. A five-state hidden Markov model was created, capable of detecting chronic seizures with $95.7 \%$ sensitivity and $98.9 \%$ specificity. It was also able to detect early and late tonic firing activities that correlate with the intracellular whole-cell dynamics prior to chronic seizure onset. Such a model would potentially allow the researchers to decipher possible sequence of distinct dynamic modes leading to seizure onsets.

\section{Acknowledgements}

The project described was supported by Grant Number P20RR016456 from the National Center for Research Resources. The authors would also like to acknowledge the Canadian Institutes of Health Research (CIHR), the Natural Science and Engineering Research Council (NSERC) of Canada and the Louisiana Board of Regents (LEQSF(2007-10)RD-A-20) for their financial support, as well as Dr. Geoffrey Hinton from the University of Toronto for his insightful feedbacks.

\section{Author details}

${ }^{1}$ Biomedical Engineering Department, Louisiana Tech University, Ruston, Louisiana, USA. ${ }^{2}$ Toronto Western Research Institute, University of Toronto, Toronto, Ontario, Canada. ${ }^{3}$ Institute of Biomaterials and Biomedical Engineering, University of Toronto, Toronto, Ontario, Canada.

\section{Authors' contributions}

AWLC and SOT carried out the data analysis and drafted the manuscript. PLC supervised and MD and MC performed the data acquisition. AWLC and BLB participated in the design of the study. All authors read and approved the current manuscript.

\section{Competing interests}

The authors declare that they have no competing interests.

Received: 26 October 2010 Accepted: 19 April 2011 Published: 19 April 2011

\section{References}

1. Milton J, Black D: Dynamic diseases in neurology and psychiatry. Chaos 1995, 5:8-13.

Shin C, McNamara J: Mechanism of epilepsy. Ann Rev Med 1994, 45:379-389.

3. Chiu AW, Jahromi SS, Khosravani H, Carlen PL, Bardakjian BL: The effects of high-frequency oscillations in hippocampal electrical activities on the classification of epileptiform events using artificial neural networks. J Neural Eng 2006, 3:9-20.

4. Lasztoczi B, Antal K, Nyikos L, Emri Z, Kardos J: High-frequency synaptic input contributes to seizure initiation in the low-[Mg2+] model of epilepsy. European Journal of Neuroscience 2004, 19:1361-1372.

5. Worrell GA, Parish L, Cranstoun SD, Jonas R, Baltuch G, Litt B: High-frequency oscillations and seizure generation in neocortical epilepsy. Brain 2004, 127:1496-1506.

6. Chiu AW, Daniel S, Khosravani H, Carlen PL, Bardakjian BL: Prediction of seizure onset in an in-vitro hippocampal slice model of epilepsy using Gaussian-based and wavelet-based artificial neural networks. Ann Biomed Eng 2005, 33:798-810.

7. Chiu AW, Kang EE, Derchansky M, Carlen PL, Bardakjian BL: Online prediction of onsets of seizure-like events in hippocampal neural networks using wavelet artificial neural networks. Ann Biomed Eng 2006, 34:282-294. 
8. Adeli H, Ghosh-Dastidar S, Dadmehr N: A wavelet-chaos methodology for analysis of EEGs and EEG subbands to detect seizure and epilepsy. IEEE Transactions on Biomedical Engineering 2007, 54:205-211.

9. Khan YU, Gotman J: Wavelet based automatic seizure detection in intracerebral electroencephalogram. Clin Neurophysiol 2003, 114:898-908.

10. Subasi A, Alkan A, Koklukaya E, Kiymik MK: Wavelet neural network classification of EEG signals by using AR model with MLE preprocessing. Neural Netw 2005, 18:985-997.

11. Osorio I, Frei MG, Wilkinson SB: Real-time automated detection and quantitative analysis of seizures and short-term prediction of clinical onset. Epilepsia 1998, 39:615-627.

12. Grewal S, Gotman J: An automatic warning system for epileptic seizures recorded on intracerebral EEGs. Clinical Neurophysiology 2005, 116:2460-2472.

13. Webber WR, Lesser RP, Richardson RT, Wilson K: An approach to seizure detection using an artificial neural network (ANN). Electroencephalogr Clin Neurophysiol 1996, 98:250-272.

14. Pradhan N, Sadasivan PK, Arunodaya GR: Detection of seizure activity in EEG by an artificial neural network: a preliminary study. Comput Biomed Res 1996, 29:303-313.

15. Kiymik MK, Subasi A, Ozcalik HR: Neural networks with periodogram and autoregressive spectral analysis methods in detection of epileptic seizure. J Med Syst 2004, 28:511-522.

16. Kim H, Rosen J: Epileptic seizure detection - an AR model based algorithm for implantable device. Conf Proc IEEE Eng Med Biol Soc 1:5541-5544.

17. Nandan M, Talathi SS, Myers SM, Ditto WL, Khargonekar PP, Carney PR: Support vector machines for seizure detection in an animal model of chronic epilepsy. Journal of Neural Engineering 2010, 7:036001.

18. Shoeb A, Edwards H, Connolly J, Bourgeois B, Treves T, Guttag J: Patient-specific seizure onset detection. Epilepsy \& Behavior 2004, 5:483-498.

19. Gabor AJ: Seizure detection using a self-organizing neural network: Validation and comparison with other detection strategies. Electroencephalography and Clinical Neurophysiology 1998, 107:27-32.

20. Karayiannis NB, Mukherjee A, Glover JR, Ktonas PY, Frost JD Jr, Hrachovy RA, Mizrahi EM: Detection of pseudosinusoidal epileptic seizure segments in the neonatal EEG by cascading a rule-based algorithm with a neural network. IEEE Trans Biomed Eng 2006, 53:633-641.

21. Guerrero-Mosquera C, Trigueros AM, Franco Jl, Navia-Vazquez A: New feature extraction approach for epileptic EEG signal detection using time-frequency distributions. Med Biol Eng Comput 48:321-330

22. Guo L, Rivero D, Pazos A: Epileptic seizure detection using multiwavelet transform based approximate entropy and artificial neural networks. J Neurosci Methods 193:156-163.

23. Ocak H: Automatic detection of epileptic seizures in EEG using discrete wavelet transform and approximate entropy. Expert Systems with Applications 2009, 36:2027-2036.

24. Perez Velazquez JL, Khosravani H, Lozano A, Bardakjian BL, Carlen PL, Wennberg R: Type III intermittency in human partial epilepsy. European Journal of Neuroscience 1999, 11:2571-2576.

25. Rabiner LR: A tutorial on hidden Markov models and selected applications in speech recognition. Proceedings of the IEEE 1989, 77:257-286.

26. Bishop CM: Pattern recognition and machine learning New York: Springer; 2006

27. Sunderam S, Osorio I, Frei MG, JF W: Stochastic modeling and prediction of experimental seizures in SpragueDawley rats. J Clin Neurophysiol 2001, 18:275-282.

28. Wong $S$, Gardner AB, Krieger AM, Litt B: A stochastic framework for evaluating seizure prediction algorithms using hidden Markov models. J Neurophysiol 2007, 97:2525-2532.

29. MacKay D: Bayesian methods for backpropagation networks Springer; 1994.

30. Akaike H: A new look at the statistical model identification. IEEE Transactions on Automatic Control 1974, AC19:716-723.

31. Burnham KP, Anderson DR: Model Selection and Multi-Model Inference: A Practical Information-Theoretic Approach Springer; 2002.

32. Talathi SS, Hwang DU, Spano ML, Simonotto J, Furman MD, Myers SM, Winters JT, Ditto WL, Carney PR: Nonparametric early seizure detection in an animal model of temporal lobe epilepsy. Journal of Neural Engineering 2008, 5:85-98.

33. Rafiq A, DeLorenzo RJ, Coulter DA: Generation and propagation of epileptiform discharges in a combined entorhinal cortex/hippocampal slice. J Neurophysiol 1993, 70:1962-1974.

34. Rafiq A, Zhang YF, DeLorenzo RJ, Coulter DA: Long-duration self-sustained epileptiform activity in the hippocampalparahippocampal slice: a model of status epilepticus. J Neurophysiol 1995, 74:2028-2042

35. Derchansky M, Jahromi SS, Mamani M, Shin DS, Sik A, Carlen PL: Transition to seizures in the isolated immature mouse hippocampus: a switch from dominant phasic inhibition to dominant phasic excitation. J Physiol 2008 , 586:477-494

36. Mody I, Lambert JD, Heinemann U: Low extracellular magnesium induces epileptiform activity and spreading depression in rat hippocampal slices. J Neurophysiol 1987, 57:869-888.

37. Blanco S, D'Attellis CE, Isaacson SI, Rosso OA, Sirae RO: Time-frequency analysis of electroencephalogram series. II. Gabor and wavelet transforms. Physical Review E - Statistical Physics, Plasmas, Fluids, and Related Interdisciplinary Topics 1996, 54:6661-6672.

38. Mallat S: A theory for multiresolution signal decomposition: the wavelet representation. IEEE transactions on pattern analysis and machine intelligence 1989, 11:674-693.

39. Rosso OA, Martin MT, Figliola A, Keller K, Plastino A: EEG analysis using wavelet-based information tools. J Neurosci Methods 2006, 153:163-182

40. Bilmes JA: A gentle tutorial on the EM algorithm and its application to parameter estimation for Gaussian mixture and hidden Markov models. Int Comput Sci Inst (ICSI) Tech Rep ICSI-TR-97-021 Berkeley, CA; 1997.

41. Peng H, Long F, Ding C: Feature selection based on mutual information: criteria of max-dependency, maxrelevance, and min-redundancy. IEEE Transactions on Pattern Analysis and Machine Intelligence 2005, 27:1226-1238.

42. Schiff SJ, Sauer T, Kumar R, Weinstein SL: Neuronal spatiotemporal pattern discrimination: the dynamical evolution of seizures. Neuroimage 2005, 28:1043-1055. 
43. Rosenstein MT, Collins JJ, Luca CJD: A practical method for calculating largest Lyapunov exponents from small data sets. Physica D 1993, 65:117-134.

44. Lasztoczi B, Kardos J: Cyclothiazide prolongs low [Mg2+]-induced seizure-like events. J Neurophysiol 2006, 96:3538-3544.

45. Khosravani H, Carlen PL, Velazquez JL: The control of seizure-like activity in the rat hippocampal slice. Biophys J 2003, 84:687-695.

46. Mormann F, Andrzejak RG, Kreuz T, Rieke C, David P, Elger CE, Lehnertz K: Automated detection of a preseizure state based on a decrease in synchronization in intracranial electroencephalogram recordings from epilepsy patients. Phys Rev E Stat Nonlin Soft Matter Phys 2003, 67:021912.

47. Alkan A, Koklukaya E, Subasi A: Automatic seizure detection in EEG using logistic regression and artificial neural network. Journal of Neuroscience Methods 2005, 148:167-176.

48. Kuhlmann L, Burkitt AN, Cook MJ, Fuller K, Grayden DB, Seiderer L, Mareels IM: Seizure detection using seizure probability estimation: comparison of features used to detect seizures. Ann Biomed Eng 2009, 37:2129-2145.

49. Tezel G, özbay Y: A new approach for epileptic seizure detection using adaptive neural network. Expert Systems with Applications 2009, 36:172-180.

50. Aarabi A, Fazel-Rezai R, Aghakhani Y: A fuzzy rule-based system for epileptic seizure detection in intracranial EEG. Clinical Neurophysiology 2009, 120:1648-1657.

51. Russell $M$, Moore R: Explicit variable duration hidden markov models for automatic speech recognition. Proc of ICASSP 1985, 5-8.

52. Gadi H, Moller DW, Valiante TA, Andrade D, Chiu AW: Spatial time-Frequency Analysis and Non-parametric Classification of Human IEEG Recordings Using HMM. BMES Annual Fall Meeting 2008.

doi:10.1186/1475-925X-10-29

Cite this article as: Chiu et al:: Wavelet-based Gaussian-mixture hidden Markov model for the detection of multistage seizure dynamics: A proof-of-concept study. BioMedical Engineering OnLine 2011 10:29.

\section{Submit your next manuscript to BioMed Central and take full advantage of:}

- Convenient online submission

- Thorough peer review

- No space constraints or color figure charges

- Immediate publication on acceptance

- Inclusion in PubMed, CAS, Scopus and Google Scholar

- Research which is freely available for redistribution 